\title{
Co-Optimization of Communication and Motion Planning of a Robotic Operation under Resource Constraints and in Fading Environments
}

\author{
Yuan Yan and Yasamin Mostofi
}

\begin{abstract}
We consider the scenario where a robot is tasked with sending a fixed number of given bits of information to a remote station, in a limited operation time, as it travels along a pre-defined trajectory, and while minimizing its motion and communication energy costs. We propose a co-optimization framework that allows the robot to plan its motion speed, transmission rate and stop time, based on its probabilistic prediction of the channel quality along the trajectory. We show that in order to save energy, the robot should move faster (slower) and send less (more) bits at the locations that have worse (better) predicted channel qualities. We furthermore prove that if the robot must stop, it should then stop only once and at the location with the best predicted channel quality. We also prove some properties for two special scenarios: the heavy-task load and the light-task load cases. We also propose an additional stop-time online adaptation strategy to further fine tune the stop location as the robot moves along its trajectory and measures the true value of the channel. Finally, our simulation results show that our proposed framework results in a considerable performance improvement.
\end{abstract}

Index Terms-Communication and motion co-optimization, realistic communication channels, energy optimization, probabilistic channel predication.

\section{INTRODUCTION}

In recent years, considerable progress has been made in the area of mobile sensor networks [2] and networked robotic systems [3]-[6]. In order to truly realize the full potential of these systems, an integrative approach to both communication and navigation issues is needed. Recently, such communicationaware navigation strategies have started to attract considerable attention [7]-[10].

In practice, energy resource of a mobile robot is typically very limited. Thus, a robot needs to efficiently plan the usage of its limited energy for its motion, communication, sensing and computation during the operation. Among these, it is reported that motion and communication are two major consumers [11]. This is the main motivation of the work proposed here. More specifically, in this paper we show how the robot can co-plan its communication and motion strategies

Manuscript received January 28, 2012; revised July 18, 2012; accepted November 9, 2012. The associate editor coordinating the review of this paper and approving it for publication was G. Bianchi.

This work is supported in part by the NSF CAREER award \# 0846483 and by the NSF award \# 0812338. A small part of this work overlaps with its conference version [1]

The authors are with the Department of Electrical and Computer Engineering, University of California Santa Barbara, CA 93106, USA (e-mail: \{yuanyan,ymostofi\}@ece.ucsb.edu).

Digital Object Identifier 10.1109/TWC.2012.12-0138. in order to minimize its total energy consumption, including both the communication and motion energy costs.

While individual optimization of communication and motion energy consumption has been heavily but separately explored in the communications/networking and robotics literature [12], [13], co-optimization of communication and motion energy consumption has received little attention so far. In [11], the authors propose an efficient algorithm to find the path that minimizes the motion and communication energy costs. However, simplified path loss models are utilized to model the communication channels. In [14], we design an optimum control law to minimize the total motion and communication costs for a robotic router network.

In this paper, we aim to design both the motion and communication strategies of a networked robotic operation, under time constraint and in realistic fading environments. More specifically, we consider a scenario where a robot needs to transmit a given number of bits of information to a fixed station, under time constraint, and as it moves along a predefined trajectory. The goal of the robot is to accomplish its task while minimizing the total motion and communication energy costs of the whole operation. We are interested in mathematically understanding when/to what extend it is beneficial for the robot to spend its energy on motion and when/to what extend it is more beneficial to spend its energy on communication (or when to do both).

Statement of Contribution: We propose a motion and communication co-optimization framework that allows the robot to schedule its motion speed, transmission rate and stop time along the pre-defined trajectory, while minimizing its overall energy consumption and satisfying a target Bit Error Rate (BER). Such a planning approach, in realistic fading environments, requires an assessment of the link qualities at places over the pre-defined trajectory that have not yet been visited by the robot. We show how our previously-proposed probabilistic channel prediction framework of [15], [16] allows the robot to assess the shadowing and path loss components of the channel over the trajectory and plan its motion and communication strategies accordingly. In particular, we prove that in order to save energy, the robot should move faster (slower) and send fewer (more) bits at the locations that have worse (better) predicted channel qualities. We furthermore prove that if the robot must stop, it should then stop only once and at the location with the best predicted channel quality. We also mathematically characterize two special scenarios, 
namely the heavy-task load and the light-task load cases, in order to have a better understanding of the optimum strategy. For the heavy-task load case, we prove that it is optimum for the robot to move quickly to the sub-trajectory with the best predicted channel quality and spend most of its time budget there, in order to save communication energy. On the other hand, if the robot has a light load, it should move at almost a constant speed along the entire trajectory in order to save motion energy.

The aforementioned optimization framework is a one-time planning at the beginning of the operation. As the robot moves along the trajectory, it can measure the true value of the channel and fine tune its strategy accordingly. Along this line, we also propose an additional stop-time online adaptation strategy to further optimize the stop location as the robot moves along its trajectory and measures the true value of the channel. For this case, we show that the problem can be posed as a nested form of multi-stage stochastic program, whose global optimum solution we mathematically characterize. Finally, we show how to extend our results to the case of online data gathering, where the robot needs to collect information from a number of points of interest along its trajectory and transmit them to the remote station. Our simulation results show that our proposed framework results in a considerable performance improvement. To the best of our knowledge, such co-optimization of communication and motion energy consumption, based on a probabilistic prediction of wireless channels, has not been proposed before.

The rest of the paper is organized as follows. Section II describes the motion and communication models, and briefly discusses the probabilistic channel assessment framework of [15], [16]. Section III presents our proposed optimization framework, based on the probabilistic assessment of shadowing and path loss terms, and proves a number of properties of the optimum solution. Section IV then shows how the robot can further adapt its strategy online as it travels along its trajectory and measures the true value of the channel. Section $\mathrm{V}$ shows the performance of the proposed framework in a simulation environment. Finally, in Section VI, we briefly show how our framework can be extended to the case of online data gathering. We conclude in Section VII.

\section{Problem Setup}

Consider the scenario where a robot is tasked with sending a fixed number of a priori-given bits of information to a remote station, in a limited operation time, and as it travels along a pre-defined trajectory $\mathcal{T}$ with length $L$. The predefined trajectory could be the only feasible path due to the environmental constraints such as obstacles. For instance, in a cluttered indoor office navigation problem, the only path from point A to point B may be across certain hallways. In Section VI where we furthermore have online sensing, the pre-defined path could be the path dictated due to sensing constraints. In [17], the authors mention that in ocean sampling, the path is typically pre-defined. Furthermore, autonomous aircrafts may be restricted to fly along a particular trajectory to stay away from commercial air traffic or avoid detection by an adversary

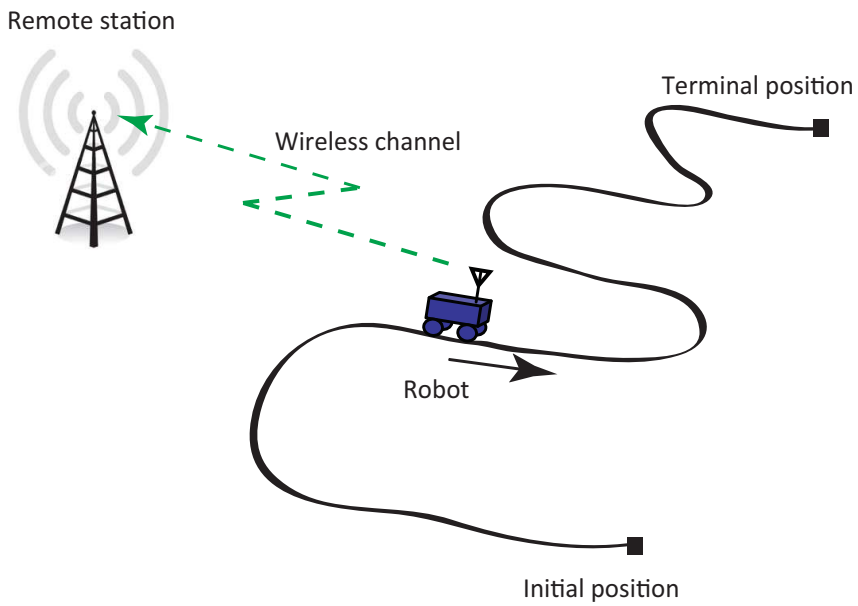

Fig. 1. The robot needs to transmit a number of given bits to the remote station, under minimum energy cost (including both motion and communication energy consumption), while traveling along a pre-defined trajectory, and under a given time budget.

[17]. ${ }^{1}$ Then, the goal of the robot is to successfully transmit the given information while minimizing the total energy consumption, which includes both motion and communication energy costs. Fig. 1 shows an example of the considered scenario. The robot starts from an initial position, follows the direction of $\mathcal{T}$, and transmits the needed information to the remote station before reaching its terminal position. In order to minimize the total energy cost, the robot needs to properly plan its motion speed/possible stop times, and schedule the transmission of the bits, based on the channel quality along the trajectory. To do so, however, the robot needs to have an assessment of the channel to the remote station along $\mathcal{T}$ before traversing it. In this section, we first summarize the probabilistic channel assessment framework that enables the robot to assess the channel at unvisited locations along its trajectory, based on a small number of channel measurements [15], [16]. We then introduce the motion and communication cost models that are used in the paper.

\section{A. Probabilistic Modeling and Prediction of a Wireless Chan- nel}

As shown in the communication literature [19], received Channel to Noise Ratio (CNR) can be modeled as a multiscale random process with three major components: path loss, shadow fading (shadowing) and multipath fading.

Let $\theta=\left[\begin{array}{ll}\alpha & n\end{array}\right]^{\mathrm{T}}, \xi_{\mathrm{dB}}, \beta$ and $\rho_{\mathrm{dB}}$ denote the vector of path loss parameters ( $n$ is the path loss exponent and $\alpha$ is the offset), standard deviation of shadowing, decorrelation distance of shadowing, and standard deviation of multipath fading respectively. Let $Y$ represent the stacked vector of $m$ a priori-gathered CNR measurements (in the $\mathrm{dB}$ domain) that are collected in the same environment, and $\mathcal{Q}=\left\{q_{1}, \cdots, q_{m}\right\}$ denote the corresponding positions. Then, a Gaussian random variable, $\Upsilon_{\mathrm{dB}}(q)$, with the

\footnotetext{
${ }^{1}$ In this paper, we solely focus on the communication and motion coplanning along a fixed path, in order to have a good understanding on how communication and motion affect each other when they are planned together However, co-planning the path itself can also be integrated into the whole framework design and is a subject of our future work. Readers are referred to our previous work [18] for solving the path planning problems in other contexts.
} 
mean of $\bar{\Upsilon}_{\mathrm{dB}}(q)=\mathbb{E}\left\{\Upsilon_{\mathrm{dB}}(q) \mid Y, \hat{\theta}, \hat{\beta}, \hat{\xi}_{\mathrm{dB}}, \hat{\rho}_{\mathrm{dB}}\right\}=H_{q} \hat{\theta}+$ $\Psi^{\mathrm{T}}(q) \Phi^{-1}\left(Y-H_{\mathcal{Q}} \hat{\theta}\right)$ and variance of $\Sigma(q)=\mathbb{E}\left\{\left(\Upsilon_{\mathrm{dB}}(q)-\right.\right.$ $\left.\left.\bar{\Upsilon}_{\mathrm{dB}}(q)\right)^{2} \mid Y, \hat{\theta}, \hat{\beta}, \hat{\xi}_{\mathrm{dB}}, \hat{\rho}_{\mathrm{dB}}\right\}=\hat{\xi}_{\mathrm{dB}}^{2}+\hat{\rho}_{\mathrm{dB}}^{2}-\Psi^{\mathrm{T}}(q) \Phi^{-1} \Psi(q)$ can best characterize the CNR (in the $\mathrm{dB}$ domain) at an unvisited

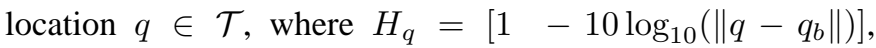
$H_{\mathcal{Q}}=\left[\mathbf{1}_{m}-D_{\mathcal{Q}}\right], \mathbf{1}_{m}$ represents the $m$-dimensional vector of all ones and $q_{b}$ is the position of the remote station. Furthermore, $D_{\mathcal{Q}}=\left[10 \log _{10}\left(\left\|q_{1}-q_{b}\right\|\right) \cdots 10 \log _{10}\left(\left\|q_{m}-q_{b}\right\|\right)\right]^{\mathrm{T}}$, $\Phi=\Omega+\hat{\rho}_{\mathrm{dB}}^{2} I_{m}$ with $\Omega$ denoting a matrix with entries $[\Omega]_{i, j}=\hat{\xi}_{\mathrm{dB}}^{2} \exp \left(-\left\|q_{i}-q_{j}\right\| / \hat{\beta}\right)$ for $i, j \in\{1, \cdots, m\}$, and $\Psi(q)=\left[\hat{\xi}_{\mathrm{dB}}^{2} \exp \left(-\left\|q-q_{1}\right\| / \hat{\beta}\right) \cdots \hat{\xi}_{\mathrm{dB}}^{2} \exp \left(-\left\|q-q_{m}\right\| / \hat{\beta}\right)\right]^{\mathrm{T}}$. The symbol ${ }^{\wedge}$ represents the estimation of the corresponding model parameters based on the a priori samples. See [15], [16] for more details on the estimation of the underlying parameters and the performance of this framework with real data and in different environments.

\section{B. Spectral Efficiency and Communication Energy Model}

In this paper, we assume MQAM modulation for the communication between the robot and the remote station. Then, we have the following approximated expression [19]: $p_{b} \approx 0.2 \exp \left(-1.5 /(M-1) P_{\mathrm{T}} \gamma\right)$, where $p_{b}$ is the Bit Error Rate (BER), $M$ represents the modulation constellation size, $P_{\mathrm{T}}$ denotes the communication transmission power and $\gamma$ is the CNR. This approximation is tight (within $1 \mathrm{~dB}$ ) when $0 \mathrm{~dB}<10 \log _{10}\left(P_{\mathrm{T}} \gamma\right)<30 \mathrm{~dB}$ [19]. Then, given a target $\mathrm{BER}, p_{b, \mathrm{th}}$, the minimum required transmit power is $P_{\mathrm{T}}=(M-1) /(K \gamma)=\left(2^{R}-1\right) /(K \gamma)$, where $K=$ $-1.5 / \ln \left(5 p_{b, \mathrm{th}}\right)$ and $R=\log _{2}(M)$ denotes the spectral efficiency.

\section{Motion Energy Model}

In this paper, we assume that the robot uses a DC motor for its motion. Based on the permanent magnet DC motor model, the motion power can be characterized as follows [13]: $P_{\mathrm{M}}=\kappa_{1} u^{2}+\kappa_{2} u+\kappa_{3}$ for $u \leq u_{\max }$, where $P_{\mathrm{M}}$ is the motion power, $u$ and $u_{\max }$ denote the velocity of the robot and its upper bound respectively, and $\kappa_{1}, \kappa_{2}$ and $\kappa_{3}$ are positive constants depending on the parameters of the motor, external load and the mechanical transmission system of the robot. This motion power model does not consider the impact of acceleration since it is negligible for many DC motors [13]. Consider the case where it takes $t$ seconds for the robot to travel a fixed length $l$ at a constant velocity of $u$. Then, we have the following total energy consumption: $E_{\mathrm{M}}=\underbrace{\frac{\kappa_{1} l^{2}}{t}+\kappa_{3} t}_{\widetilde{E}_{\mathrm{M}}}+\kappa_{2} l$ for $t \geq l / u_{\max }$. Since we have a fixed trajectory, the variable $u$ only affects $\widetilde{E}_{\mathrm{M}}$. Hence, we use $\widetilde{E}_{\mathrm{M}}$ instead of $E_{\mathrm{M}}$ to denote the motion energy cost when formulating our optimization framework in Section III. Note that the first term of $E_{\mathrm{M}}$ is inversely proportional to $t$ while the second term is linear in $t$. Hence, the motion energy cost becomes large if the robot moves too fast or too slow, with the minimum achieved at $t= \begin{cases}\sqrt{\kappa_{1} / \kappa_{3} l} & \text { if } u_{\max }>\sqrt{\kappa_{3} / \kappa_{1}} \\ l / u_{\max } & \text { if } u_{\max } \leq \sqrt{\kappa_{3} / \kappa_{1}}, \text { or equivalently at }\end{cases}$ $u=\left\{\begin{array}{ll}\sqrt{\kappa_{3} / \kappa_{1}} & \text { if } u_{\max }>\sqrt{\kappa_{3} / \kappa_{1}} \\ u_{\max } & \text { if } u_{\max } \leq \sqrt{\kappa_{3} / \kappa_{1}}\end{array}\right.$.

III. CO-Optimization OF Motion AND

COMMUNICATION COSTS BASED ON THE LEARNING OF

THE PATH LOSS AND SHADOWING COMPONENTS

We divide the whole trajectory into $N_{\mathrm{SH}}$ sub-trajectories, $\mathcal{T}_{i} \mathrm{~s}$, for $i \in\left\{1,2, \cdots, N_{\mathrm{SH}}\right\}$, each with length $l_{i}$. We assume small enough $l_{i}$ such that the path loss and shadowing components of the channel can be assumed constant over each $\mathcal{T}_{i}$. The length $l_{i}$ then depends on the length over which the channel can be considered stationary. To consider the most general case, we further allow $l_{i}$ s to be different from each other in order to account for the cases where the trajectory spans over a large area with changing environmental features (such as from indoor to outdoor), resulting in different stationary lengths in different parts of the trajectory. Note that the channel is still space-varying over each $\mathcal{T}_{i}$ due to multipath fading.

In this section, we show how the robot can co-plan its motion and communication, based on its prediction of the path loss and shadowing components of the channel over the subtrajectories. More specifically, we mathematically characterize some of the properties of the optimum solution. Our one-time co-optimization happens at the beginning of the operation. In Section IV, we then show how the robot can furthermore fine tune its strategy through online adaptation to the multipath component, as it moves along each sub-trajectory and measures the true value of the channel.

\section{A. Planning Based on the Prediction of the Path Loss and Shadowing Components}

Assume that the robot starts with a number of initial CNR measurements collected a priori in the same environment where it is operating. It can then use the aforementioned probabilistic channel assessment framework of Section II-A to predict the path loss and shadowing components of the received CNR over each sub-trajectory $\mathcal{T}_{i}$. Let $q_{i} \in \mathcal{T}_{i}$ represent a point along $\mathcal{T}_{i}$. Then the $\mathrm{CNR}$ at $\mathcal{T}_{i}$ can be assessed as follows: $\Upsilon_{\mathrm{dB}}\left(q_{i}\right) \sim \mathcal{N}\left(\bar{\Upsilon}_{\mathrm{dB}}\left(q_{i}\right), \Sigma\left(q_{i}\right)\right)$. Based on the aforementioned communication and motion energy models, we propose the following optimization framework to minimize the average total energy consumption (averaged over the distribution of the channel), where $\widetilde{R}_{i} \geq 0, \widetilde{t}_{\mathrm{st}, i} \geq 0$, $\widetilde{t}_{\mathrm{tr}, i} \geq 0$ and $\widetilde{t}_{\mathrm{mo}, i} \geq l_{i} / u_{\mathrm{max}}$ :

$$
\begin{aligned}
& \min \tilde{\mathcal{J}}_{\mathrm{SH}}=\sum_{i=1}^{N_{\mathrm{SH}}} \underbrace{\frac{2^{\widetilde{R}_{i}}-1}{K} \mathbb{E}\left\{\frac{1}{\Upsilon_{i}}\right\} \widetilde{t}_{\mathrm{tr}, i}}_{E_{\mathrm{C}, i}: \text { ave. comm. energy cost along }}
\end{aligned}
$$

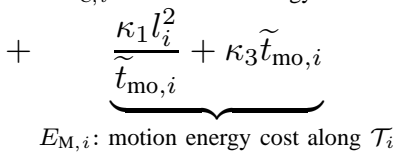

$$
\begin{aligned}
& \text { s.t. } \quad \sum_{i=1}^{N_{\mathrm{SH}}} \widetilde{t}_{\mathrm{mo}, i}+\widetilde{t}_{\mathrm{st}, i} \leq T, \quad \sum_{i=1}^{N_{\mathrm{SH}}} \widetilde{R}_{i} \widetilde{t}_{\mathrm{tr}, i}=Q / B, \\
& \widetilde{t}_{\mathrm{tr}, i} \leq \widetilde{t}_{\mathrm{mo}, i}+\widetilde{t}_{\mathrm{st}, i}, \quad \forall i \in\left\{1, \cdots, N_{\mathrm{SH}}\right\},
\end{aligned}
$$

where the unknown variables to solve for are $\widetilde{R}_{i}, \widetilde{t}_{\mathrm{tr}, i}, \widetilde{t}_{\mathrm{mo}, i}$ and $\widetilde{t}_{\mathrm{st}, i}$, which denote the spectral efficiency, transmit time, 
motion time and stop time that are assigned to each $\mathcal{T}_{i}$ respectively. ${ }^{2}$ Furthermore, $\Upsilon_{i}$ represents the non-dB version of $\Upsilon_{\mathrm{dB}}\left(q_{i}\right)$, i.e. $\Upsilon_{i}=\Upsilon\left(q_{i}\right)=10^{\Upsilon_{\mathrm{dB}}\left(q_{i}\right) / 10}, T \geq L / u_{\max }$ is the given operation time budget, $0<Q<\infty$ is the total number of bits that needs to be sent, and $B$ is the given fixed bandwidth. ${ }^{3}$ As mentioned in Section II-B, the required target BER is part of $K: K=-1.5 / \ln \left(5 p_{b, \text { th }}\right)$. In the formulation of (1), we assume that the robot travels at a constant speed along each sub-trajectory. Then, the motion energy cost can be characterized by using $\widetilde{E}_{\mathrm{M}}$.

Our optimization problem of (1) plans the motion speed/stop time of the robot (available time budget) and schedules the transmission of the given bits along each subtrajectory, while minimizing the average total energy cost and satisfying the time budget and target BER. Note that motion energy can be minimized by using the velocity mentioned in Section II-C. Thus, our co-optimization framework essentially plans the motion strategy, i.e. possibly costing more motion energy, to help save communication energy, resulting in an overall energy cost reduction. Since the robot can incur a large amount of motion energy if it moves too slowly, we introduced the stop time variables $\widetilde{t}_{\mathrm{st}, i} \mathrm{~s}$ in (1), in order to allow the robot to stop during the operation if needed. Then, the total time that the robot can spend along $\mathcal{T}_{i}$ is $\widetilde{t}_{\mathrm{mo}, i}+\widetilde{t}_{\mathrm{st}, i}$, which includes both the motion and stop time durations. Hence, the transmit time $\widetilde{t}_{\mathrm{tr}, i}$ along $\mathcal{T}_{i}$ should always be smaller than or equal to $\widetilde{t}_{\mathrm{mo}, i}+\widetilde{t}_{\mathrm{st}, i}: \widetilde{t}_{\mathrm{tr}, i} \leq \widetilde{t}_{\mathrm{mo}, i}+\widetilde{t}_{\mathrm{st}, i}$. In Section IV, we will further show where the robot should stop along the sub-trajectory as it measures the real value of CNR.

Remark 1: In this paper, when we say that $\mathcal{T}_{i}$ has a good (or bad) predicted channel quality, we mean that $\mathbb{E}\left\{1 / \Upsilon_{i}\right\}$ is small (or large). From Section II-A, $\Upsilon_{i}$ is a lognormal random variable. Then, it is straightforward to show that, $\mathbb{E}\left\{1 / \Upsilon_{i}\right\}=$ $\exp \left((\ln 10 / 10)^{2} \Sigma_{i} / 2\right) / \bar{\Upsilon}_{i}$, where $\bar{\Upsilon}_{i}=10^{\bar{\Upsilon}_{\mathrm{dB}}\left(q_{i}\right) / 10}$ and $\Sigma_{i}=\Sigma\left(q_{i}\right)$. Therefore, the robot has a better predicted channel quality at $\mathcal{T}_{i}$ if the corresponding predicted mean value $\left(\bar{\Upsilon}_{i}\right)$ is relatively large and/or the prediction error variance $\left(\Sigma_{i}\right)$ is relatively small. This is, in particular, a good measure of assessing the link quality. It not only takes into account the predicted channel value $\left(\bar{\Upsilon}_{i}\right)$ but also considers how much the robot trusts its prediction $\left(\Sigma_{i}\right)$. This becomes important in the optimization of resource allocation so the robot does not allocate as much resources to the places where it has a high prediction error variance.

There is no closed-form solution for (1). However, we can still characterize certain properties of the optimum solution, as we show next. In what follows, we use superscript $\star$ to represent the optimum solution (or value) of the corresponding optimization problem. First, we show that (1) can be simplified to another optimization problem with the same optimum value.

Lemma 1: $g(t)=\left(2^{C / t}-1\right) t$ is a non-increasing function

${ }^{2}$ In practice, $\widetilde{R}_{i}$ s should have certain integer constraints to ensure a proper modulation [19]. In this paper, we do not consider such constraints for the sake of mathematical analysis. However, these constraints can be added to (1), resulting in a mixed integer program. Alternatively, they can be applied after solving this optimization problem.

${ }^{3}$ Note that (1) is infeasible if $T<L / u_{\max }$. Other feasibility issues can arise if maximum spectral efficiency and/or maximum transmit power are considered. While we do not consider these constraints due to page limitation, we note that similar results can be derived. of $t$ for $t>0$.

Proof: The lemma can be easily confirmed by finding the first and second-order derivatives of $g(t)$.

Lemma 2: The optimum solution of (1) is the same as the optimum solution of the following optimization problem for $R_{i} \geq 0, t_{\mathrm{st}, i} \geq 0$ and $t_{\mathrm{mo}, i} \geq l_{i} / u_{\max }, \forall i \in\left\{1, \cdots, N_{\mathrm{SH}}\right\}$ :

$$
\begin{array}{ll}
\min \quad \mathcal{J}_{\mathrm{SH}}= & \sum_{i=1}^{N_{\mathrm{SH}}} \frac{2^{R_{i}}-1}{K} \mathbb{E}\left\{\frac{1}{\Upsilon_{i}}\right\}\left(t_{\mathrm{mo}, i}+t_{\mathrm{st}, i}\right) \\
& +\frac{\kappa_{1} l_{i}^{2}}{t_{\mathrm{mo}, i}}+\kappa_{3} t_{\mathrm{mo}, i} \\
\text { s.t. } \quad & \sum_{i=1}^{N_{\mathrm{SH}}} t_{\mathrm{mo}, i}+t_{\mathrm{st}, i} \leq T, \quad \sum_{i=1}^{N_{\mathrm{SH}}} R_{i}\left(t_{\mathrm{mo}, i}+t_{\mathrm{st}, i}\right)=Q / B .
\end{array}
$$

Proof: Note that (2) is a special case of (1) with $\widetilde{t}_{\mathrm{tr}, i}=$ $\widetilde{t}_{\mathrm{mo}, i}+\widetilde{t}_{\mathrm{st}, i}$ for all $i$, i.e. the communication transmission time is taken to be the same as the total time spent in each subtrajectory. Let $\widetilde{R}_{i}^{\star}, \widetilde{t}_{\mathrm{tr}, i}^{\star}, \widetilde{t}_{\mathrm{mo}, i}^{\star}$ and $\widetilde{t}_{\mathrm{st}, i}^{\star}$ represent the optimum solution of (1). From comparing the objective functions of (1) and (2), we can easily see that $\widetilde{\mathcal{J}}_{\mathrm{SH}}^{\star} \leq \mathcal{J}_{\mathrm{SH}}^{\star}$. We then pick a feasible solution for (2) as follows: $t_{\mathrm{mo}, i}=\widetilde{t}_{\mathrm{mo}, i}^{\star}, t_{\mathrm{st}, i}=$ $\widetilde{t}_{\mathrm{st}, i}^{\star}$, and $R_{i}=\left\{\begin{array}{ll}\widetilde{R}_{i}^{\star} \widetilde{t}_{\mathrm{tr}, i}^{\star} /\left(\widetilde{t}_{\mathrm{mo}, i}^{\star}+\widetilde{t}_{\mathrm{st}, i}^{\star}\right) & \text { if } \widetilde{R}_{i}^{\star}, \widetilde{t}_{\mathrm{tr}, i}^{\star}>0 \\ 0 & \text { otherwise }\end{array}\right.$. By using Lemma 1, we have $\left(2^{R_{i}}-1\right)\left(t_{\mathrm{mo}, i}+t_{\mathrm{st}, i}\right) \leq\left(2^{\widetilde{R}_{i}^{\star}}-1\right) \widetilde{t}_{\mathrm{tr}, i}^{\star}$, which results in $\mathcal{J}_{\mathrm{SH}}^{\star} \leq \mathcal{J}_{\mathrm{SH}}\left(R_{i}, t_{\mathrm{mo}, i}, t_{\mathrm{st}, i}\right) \leq \widetilde{\mathcal{J}}_{\mathrm{SH}}^{\star}$. Thus, we must have $\mathcal{J}_{\mathrm{SH}}^{\star}=\mathcal{\mathcal { J }}_{\mathrm{SH}}^{\star}$.

Intuitively, it always costs less communication energy if the robot sends a fixed number of bits over a longer period of time, as it can then reduce the spectral efficiency (can send with a lower rate). This is what Lemma 2 indicates. It can be seen that the optimality of (1) can be achieved only if $\widetilde{t}_{\mathrm{tr}, i}=\widetilde{t}_{\mathrm{mo}, i}+\widetilde{t}_{\mathrm{st}, i}$ for all $i$, i.e. the transmission time in each sub-trajectory is taken equal to the time spent in each subtrajectory (the maximum possible). Note that (2) also reduces the dimension of the optimization problem. Based on Lemma 2 , we can then characterize the properties of the optimum solution of (2) instead of (1) in the rest of the paper. Note that if $T=L / u_{\max }$ in (2), then $t_{\mathrm{mo}, i}=l_{i} / u_{\max }$ and $t_{\mathrm{st}, i}=0$ for all $i$, resulting in a simplified convex optimization problem which only has variable $R_{i}$. In this case, the robot does not have any freedom to plan its motion policy because of the limited time budget. The problem then becomes a spectral efficiency optimization problem, which can be characterized by using the approach in [12]. Hence, in the rest of the paper, we focus on the case where $T>L / u_{\max }$. Next, we present the following well-known theorem in optimization theory, which we use in our subsequent proofs.

Definition 1 (LICQ, [20]): The Linear Independence Constraint Qualification (LICQ) holds if the gradients of the active constraints (those that reach equality) are linearly independent.

Theorem 1 (First-Order Necessary Conditions, [20]):

Suppose that $p^{\star}$ is a local solution of a constrained optimization problem, that the objective function and the constraints are continuously differentiable, and that the LICQ holds at $p^{\star}$. Then there exists a Lagrange multiplier vector $\lambda^{\star}$, such that Karush-Kuhn-Tucker (KKT) conditions hold at $\left(p^{\star}, \lambda^{\star}\right)$. 
Then, we have the following regarding the optimum solution of (2).

Lemma 3: The constraints of (2) satisfy the LICQ at the optimum point if $T>L / u_{\max }$.

Proof: The gradients of the active constraints with respect to the variable $\left[R_{1} \cdots R_{N_{\mathrm{SH}}}\left|t_{\mathrm{mo}, 1} \cdots t_{\mathrm{mo}, N_{\mathrm{SH}}}\right| t_{\mathrm{st}, 1}\right.$ $\left.\cdots t_{\mathrm{st}, N_{\mathrm{SH}}}\right]^{\mathrm{T}}$ are as follows: $\left[\mathbf{0}_{N_{\mathrm{SH}}^{\mathrm{T}}}^{\mathrm{T}}\left|\mathbf{1}_{N_{\mathrm{SH}}}^{\mathrm{T}}\right| \mathbf{1}_{N_{\mathrm{SH}}}^{\mathrm{T}}\right]^{\mathrm{T}}$ (if the first constraint is active), $\left[t_{\mathrm{mo}, 1}+t_{\mathrm{st}, 1} \cdots t_{\mathrm{mo}, N_{\mathrm{SH}}}+\right.$ $\left.t_{\mathrm{st}, N_{\mathrm{SH}}}\left|R_{1} \cdots R_{N_{\mathrm{SH}}}\right| R_{1} \cdots R_{N_{\mathrm{SH}}}\right]^{\mathrm{T}}$ (for the second constraint), $\left[\mathbf{e}_{N_{\mathrm{SH}}}^{\mathrm{T}}(i)\left|\mathbf{0}_{N_{\mathrm{SH}}}^{\mathrm{T}}\right| \mathbf{0}_{N_{\mathrm{SH}}}^{\mathrm{T}}\right]^{\mathrm{T}}$ (if $R_{i}=0$ ), $\left[\mathbf{0}_{N_{\mathrm{SH}}}^{\mathrm{T}}\left|\mathbf{e}_{N_{\mathrm{SH}}}^{\mathrm{T}}(i)\right| \mathbf{0}_{N_{\mathrm{SH}}}^{\mathrm{T}}\right]^{\mathrm{T}}$ (if $t_{\mathrm{mo}, i}=l_{i} / u_{\max }$ ) and $\left[\mathbf{0}_{N_{\mathrm{SH}}}^{\mathrm{T}}\left|\mathbf{0}_{N_{\mathrm{SH}}}^{\mathrm{T}}\right| \mathbf{e}_{N_{\mathrm{SH}}}^{\mathrm{T}}(i)\right]^{\mathrm{T}}$ (if $t_{\mathrm{st}, i}=0$ ), where $\mathbf{e}_{N_{\mathrm{SH}}}(i)$ represents the $N_{\mathrm{SH}}$-dimensional unit vector with the $i^{\text {th }}$ entry equal to 1 , and $\mathbf{0}_{N_{\mathrm{SH}}}$ and $\mathbf{1}_{N_{\mathrm{SH}}}$ denote $N_{\mathrm{SH}^{-}}$ dimensional vectors of all 0 and 1 respectively. By assuming that $Q>0$, we have $R_{i}^{\star}>0$ for some $i \in\left\{1, \cdots, N_{\mathrm{SH}}\right\}$. Furthermore, if $T>L / u_{\max }$, then the first constraint and $t_{\mathrm{mo}, i} \geq l_{i} / u_{\max }$, for all $i$, cannot be all active at the same time. Then, all the gradients of the active constraints cannot be linearly dependent at the optimum point in this case. Therefore, the constraints in (2) satisfy the LICQ at the optimum point.

Next, we show the properties of the optimum strategy based on Theorem 1 for the co-optimization of communication and motion.

Theorem 2: The optimum motion speed $\left(u_{i}^{\star}\right)$, transmission rate $\left(R_{i}^{\star}\right)$ and stop time $\left(t_{\mathrm{st}, i}^{\star}\right)$ of (2) satisfy the following properties: if $\mathbb{E}\left\{1 / \Upsilon_{i}\right\}<\mathbb{E}\left\{1 / \Upsilon_{j}\right\}$, then $u_{i}^{\star} \leq u_{j}^{\star}$ and $R_{i}^{\star} \geq R_{j}^{\star}$ for $i, j \in\left\{1, \cdots, N_{\mathrm{SH}}\right\}$. Moreover, if $\mathbb{E}\left\{1 / \Upsilon_{i}\right\}$ is above a certain threshold, then there is no transmission in the corresponding sub-trajectory $\left(R_{i}^{\star}=0\right)$. Finally, if $t_{\mathrm{st}, i}^{\star}>0$, then $\mathbb{E}\left\{1 / \Upsilon_{i}\right\}=\min _{j \in\left\{1, \cdots, N_{\mathrm{SH}}\right\}}\left\{\mathbb{E}\left\{1 / \Upsilon_{j}\right\}\right\}$, i.e. if the robot should stop, it stops at the sub-trajectory with the best predicted channel quality.

Proof: We have the following dual function for the optimization problem of (2): $f_{\mathcal{J}_{\mathrm{SH}}}=\sum_{i=1}^{N_{\mathrm{SH}}}\left(\left(2^{R_{i}}\right.\right.$ $-1) / K) \mathbb{E}\left\{1 / \Upsilon_{i}\right\}\left(t_{\mathrm{mo}, i}+t_{\mathrm{st}, i}\right)+\kappa_{1} l_{i}^{2} / t_{\mathrm{mo}, i}+\kappa_{3} t_{\mathrm{mo}, i}+$ $\nu\left(\sum_{i=1}^{N_{\mathrm{SH}}} t_{\mathrm{mo}, i}+t_{\mathrm{st}, i}-T\right)-\lambda\left(\sum_{i=1}^{N_{\mathrm{SH}}} R_{i}\left(t_{\mathrm{mo}, i}+t_{\mathrm{st}, i}\right)-Q / B\right)-$ $\sum_{i=1}^{N_{\mathrm{SH}}} \pi_{i} R_{i}-\sum_{i=1}^{N_{\mathrm{SH}}} \epsilon_{i} t_{\mathrm{mo}, i}-\sum_{i=1}^{N_{\mathrm{SH}}} \delta_{i} t_{\mathrm{st}, i}$, where $\pi_{i}, \epsilon_{i}, \delta_{i}, \nu$ and $\lambda$ are Lagrange multipliers. From Lemma 3 , the optimum solution of (2) should satisfy the following KKT conditions:

$$
\begin{aligned}
& \frac{\partial f_{\mathcal{J}_{\mathrm{sH}}}}{\partial R_{i}}=\left(\frac{2^{R_{i}} \ln (2)}{K} \mathbb{E}\left\{\frac{1}{\Upsilon_{i}}\right\}-\lambda\right)\left(t_{\mathrm{mo}, i}+t_{\mathrm{st}, i}\right)-\pi_{i}=0, \\
& \frac{\partial f_{\mathcal{J}_{\mathrm{sH}}}}{\partial t_{\mathrm{mo}, i}}=\frac{2^{R_{i}}-1}{K} \mathbb{E}\left\{\frac{1}{\Upsilon_{i}}\right\}-\lambda R_{i}-\frac{\kappa_{1} l_{i}^{2}}{t_{\mathrm{mo}, i}^{2}}+\kappa_{3}+\nu-\epsilon_{i}=0, \\
& \frac{\partial f_{\mathcal{J}_{\mathrm{sH}}}}{\partial t_{\mathrm{st}, i}}=\frac{2^{R_{i}}-1}{K} \mathbb{E}\left\{\frac{1}{\Upsilon_{i}}\right\}-\lambda R_{i}+\nu-\delta_{i}=0, \\
& \nu\left(\sum_{i=1}^{N_{\mathrm{SH}}} t_{\mathrm{mo}, i}+t_{\mathrm{st}, i}-T\right)=0, \\
& \lambda\left(\sum_{i=1}^{N_{\mathrm{SH}}} R_{i}\left(t_{\mathrm{mo}, i}+t_{\mathrm{st}, i}\right)-Q / B\right)=0, \quad \pi_{i} R_{i}=0, \\
& \epsilon_{i}\left(t_{\mathrm{mo}, i}-l_{i} / u_{\mathrm{max}}\right)=0, \quad \delta_{i} t_{\mathrm{st}, i}=0, \\
& R_{i}, t_{\mathrm{st}, i}, \delta_{i}, \epsilon_{i}, \lambda, \nu \geq 0, \quad t_{\mathrm{mo}, i} \geq l_{i} / u_{\mathrm{max}} .
\end{aligned}
$$

Moreover, the first three KKT conditions can be further simplified as follows:

$$
\begin{aligned}
& R_{i}^{\star}= \begin{cases}\log _{2}\left(\frac{\lambda^{\star} K}{\ln (2)} / \mathbb{E}\left\{\frac{1}{\Upsilon_{i}}\right\}\right) & \text { if } i \in \mathcal{I}, \\
0 & \text { otherwise, }\end{cases} \\
& \frac{\kappa_{1} l_{i}^{2}}{t_{\mathrm{mo}, i}^{2}}=\kappa_{1} u_{i}^{\star 2}= \begin{cases}\lambda^{\star}\left(\frac{1}{\ln (2)}-R_{i}^{\star}\right)-\frac{1}{K} \mathbb{E}\left\{\frac{1}{\Upsilon_{i}}\right\} \\
+\nu^{\star}+\kappa_{3}-\epsilon_{i}^{\star} & \text { if } i \in \mathcal{I}, \\
\nu^{\star}+\kappa_{3}-\epsilon_{i}^{\star} & \text { otherwise, }\end{cases}
\end{aligned}
$$

and $\delta_{i}^{\star}=\kappa_{1} u_{i}^{\star 2}-\kappa_{3}+\epsilon_{i}^{\star}$,

where $\mathcal{I}=\left\{i \in\left\{1, \cdots, N_{\mathrm{SH}}\right\} \mid \mathbb{E}\left\{1 / \Upsilon_{i}\right\}<\lambda^{\star} K / \ln (2)\right\}$.

From (3), it can be seen that $R_{i}^{\star}=0$ if $\mathbb{E}\left\{1 / \Upsilon_{i}\right\}$ is above the threshold $\lambda^{\star} K / \ln (2)$. This means that the robot does not transmit if the predicted channel quality is below a certain level. Furthermore, for $i, j \in \mathcal{I}$, we can see that $R_{i}^{\star}>R_{j}^{\star}$ if $\mathbb{E}\left\{1 / \Upsilon_{i}\right\}<\mathbb{E}\left\{1 / \Upsilon_{j}\right\}$. In summary, we have $R_{i}^{\star} \geq R_{j}^{\star}$ if $\mathbb{E}\left\{1 / \Upsilon_{i}\right\}<\mathbb{E}\left\{1 / \Upsilon_{j}\right\}$.

Next, consider $\kappa_{1} u_{i}^{\star 2}$ in (3). It is straightforward to verify that $\kappa_{1} u_{i}^{\star 2}+\epsilon_{i}^{\star} \leq \kappa_{1} u_{j}^{\star 2}+\epsilon_{j}^{\star}$ if $\mathbb{E}\left\{1 / \Upsilon_{i}\right\}<\mathbb{E}\left\{1 / \Upsilon_{j}\right\}$. Note that the equality holds only if $i, j \in\left\{1, \cdots, N_{\mathrm{SH}}\right\} \backslash \mathcal{I}$. Suppose that $u_{i}^{\star}>u_{j}^{\star}$, then $0 \leq \epsilon_{i}^{\star}<\epsilon_{j}^{\star}$, resulting in $t_{\mathrm{mo}, j}^{\star}=$ $l_{j} / u_{\max }$, or equivalently $u_{j}^{\star}=u_{\max } \geq u_{i}^{\star}$. This contradicts the assumption that $u_{i}^{\star}>u_{j}^{\star}$. Hence, we have $u_{i}^{\star} \leq u_{j}^{\star}$ if $\mathbb{E}\left\{1 / \Upsilon_{i}\right\}<\mathbb{E}\left\{1 / \Upsilon_{j}\right\}$

Finally, suppose that $t_{\mathrm{st}, j}^{\star}>0$ and $\mathbb{E}\left\{1 / \Upsilon_{j}\right\}>$ $\min _{k \in\left\{1, \cdots, N_{\mathrm{SH}}\right\}}\left\{\mathbb{E}\left\{1 / \Upsilon_{k}\right\}\right\}$. Then there exists some $i \in \mathcal{I}$ such that $\mathbb{E}\left\{1 / \Upsilon_{i}\right\}<\mathbb{E}\left\{1 / \Upsilon_{j}\right\}$. This means that $\kappa_{1} u_{i}^{\star 2}+$ $\epsilon_{i}^{\star}<\kappa_{1} u_{j}^{\star 2}+\epsilon_{j}^{\star}$, which results in $\delta_{i}^{\star}<\delta_{j}^{\star}$. Moreover, from the KKT conditions, we know that $\delta_{j}^{\star}=0$ given $t_{\mathrm{st}, j}^{\star}>0$, which results in $\delta_{i}^{\star}<\delta_{j}^{\star}=0$. This contradicts the constraint that $\delta_{i} \geq 0$. Hence, if $t_{\mathrm{st}, i}^{\star}>0$, then we must have $\mathbb{E}\left\{1 / \Upsilon_{i}\right\}=\min _{j \in\left\{1, \cdots, N_{\mathrm{SH}}\right\}}\left\{\mathbb{E}\left\{1 / \Upsilon_{j}\right\}\right\}$.

Theorem 2 shows that the robot should move slower at the locations that have higher predicted channel qualities in order to send more bits. On the other hand, if the predicted channel qualities are low, it should then speed up to escape from these regions quickly. Also, the robot should transmit faster (slower) at the locations that have higher (lower) predicted channel qualities. If the predicted channel qualities are too low, the robot should not transmit any information. As mentioned previously, we have $\mathbb{E}\left\{1 / \Upsilon_{i}\right\}=\exp \left((\ln 10 / 10)^{2} \Sigma_{i} / 2\right) / \bar{\Upsilon}_{i}$, which depends on the predicted mean value $\left(\bar{\Upsilon}_{i}\right)$ and the prediction error variance $\left(\Sigma_{i}\right)$. Hence, the robot moves slower and transmits faster at the locations that have larger predicted mean values and/or smaller prediction error variance. Finally, Theorem 2 says that if the robot must stop, it should then stop only once and at the location with the best predicted channel quality.

Corollary 1: Let $k_{\text {best }}$ denote the index where $\mathbb{E}\left\{1 / \Upsilon_{i}\right\}$ has its minimum based on the predicted channel, i.e. $k_{\text {best }}=$ $\arg \min _{i \in\left\{1, \cdots, N_{\mathrm{SH}}\right\}}\left\{\mathbb{E}\left\{1 / \Upsilon_{i}\right\}\right\}$. If $u_{\max }>\sqrt{\kappa_{3} / \kappa_{1}}$, we have $u_{i}^{\star} \geq \sqrt{\kappa_{3} / \kappa_{1}}$ for all $i$. Moreover, if $t_{\mathrm{st}, k_{\text {best }}}^{\star}>0$, then $u_{k_{\text {best }}}^{\star}=\sqrt{\kappa_{3} / \kappa_{1}}$. If $u_{\max } \leq \sqrt{\kappa_{3} / \kappa_{1}}$, we have $u_{i}^{\star}=u_{\max }$ for all $i$, and $t_{\text {st }, k_{\text {best }}}^{\star}=T-L / u_{\text {max }}$.

Proof: From the proof of Theorem 2, we know that $\delta_{i}^{\star}=$ $\kappa_{1} u_{i}^{\star 2}-\kappa_{3}+\epsilon_{i}^{\star} \geq 0$ for all $i$. Moreover, if $t_{\mathrm{st}, k_{\text {best }}}^{\star}>0$, then we have $\delta_{k_{\text {best }}}^{\star}=0$. For the case of $u_{\max }>\sqrt{\kappa_{3} / \kappa_{1}}$, it is straightforward to verify that $\kappa_{1} u_{i}^{\star 2}-\kappa_{3}+\epsilon_{i}^{\star} \geq 0$ holds only 
if $u_{i}^{\star} \geq \sqrt{\kappa_{3} / \kappa_{1}}$. Furthermore, if $t_{\text {st, } k_{\text {best }}}^{\star}>0$, then $u_{k_{\text {best }}}^{\star}=$ $\sqrt{\kappa_{3} / \kappa_{1}}$. Similarly, for the case of $u_{\max } \leq \sqrt{\kappa_{3} / \kappa_{1}}$, the only possible solution is $u_{i}^{\star}=u_{\max }$ for all $i$, which also implies that $t_{\mathrm{st}, k_{\text {best }}}^{\star}=T-L / u_{\max }$.

Theorem 2 and Corollary 1 state that, in order to spend long enough time at the sub-trajectory where the predicted channel quality is the best, the robot stops at this location rather than reducing its speed below $\sqrt{\kappa_{3} / \kappa_{1}}$ if $u_{\max }>\sqrt{\kappa_{3} / \kappa_{1}}$ (or below $u_{\max }$ if $\left.u_{\max } \leq \sqrt{\kappa_{3} / \kappa_{1}}\right)$. As shown in Section II-C, the aforementioned velocity minimizes the motion energy cost. Hence, the robot never reduces its speed below it in order to save the total energy.

Next, we consider the resulting optimum energy consumption of the robot. Consider the motion and communication energy costs $\left(E_{\mathrm{M}, i}\right.$ and $\left.E_{\mathrm{C}, i}\right)$ as defined in (1). Define $E_{\mathrm{M}, \text { norm }, i} \triangleq E_{\mathrm{M}, i} / l_{i}=\kappa_{1} l_{i} / t_{\mathrm{mo}, i}+\kappa_{3} t_{\mathrm{mo}, i} / l_{i}$ and $E_{\mathrm{C}, \text { norm }, i} \triangleq$ $E_{\mathrm{C}, i} / l_{i}=\left(2^{R_{i}}-1\right) \mathbb{E}\left\{1 / \Upsilon_{i}\right\}\left(t_{\mathrm{mo}, i}+t_{\mathrm{st}, i}\right) /\left(l_{i} K\right)$ as the motion energy cost and the communication energy cost per unit length respectively. Then, based on the results of Theorem 2, we have the following corollary at the optimum solution, where $i, j \in\left\{1, \cdots, N_{\mathrm{SH}}\right\}$ :

Corollary 2: If $\mathbb{E}\left\{1 / \Upsilon_{i}\right\}<\mathbb{E}\left\{1 / \Upsilon_{j}\right\}$, then $E_{\mathrm{M}, \text { norm }, i}^{\star} \leq$ $E_{\mathrm{M}, \text { norm }, j}^{\star}$ and $E_{\mathrm{C}, \text { norm }, i}^{\star} \geq E_{\mathrm{C}, \text { norm }, j}^{\star}$.

Proof: From Theorem 2, we have $u_{i}^{\star} \leq u_{j}^{\star}, R_{i}^{\star} \geq R_{j}^{\star}$ and $t_{\mathrm{st}, i}^{\star} \geq t_{\mathrm{st}, j}^{\star}$ if $\mathbb{E}\left\{1 / \Upsilon_{i}\right\}<\mathbb{E}\left\{1 / \Upsilon_{j}\right\}$. Since $E_{\mathrm{C}, \text { norm }, i}$ is monotonically increasing with respect to $R_{i}$ and $t_{\mathrm{st}, i}$, and is monotonically decreasing with respect to $u_{i}$, then $E_{\mathrm{C}, \text { norm }, i}^{\star} \geq$ $E_{\mathrm{C}, \text { norm }, j}^{\star}$ if $\mathbb{E}\left\{1 / \Upsilon_{i}\right\}<\mathbb{E}\left\{1 / \Upsilon_{j}\right\}$. Moreover, from Corollary 1 , we have $u_{j}^{\star} \geq u_{i}^{\star} \geq \sqrt{\kappa_{3} / \kappa_{1}}$ if $u_{\max }>\sqrt{\kappa_{3} / \kappa_{1}}$, and $u_{j}^{\star}=u_{i}^{\star}=u_{\max }$ if $u_{\max } \leq \sqrt{\kappa_{3} / \kappa_{1}}$. Since $E_{\mathrm{M}, \text { norm }, i}$ is monotonically increasing with respect to $u_{i} \geq \sqrt{\kappa_{3} / \kappa_{1}}$, then $E_{\mathrm{M}, \text { norm }, i}^{\star} \leq E_{\mathrm{M}, \text { norm }, j}^{\star}$ if $u_{\max }>\sqrt{\kappa_{3} / \kappa_{1}}$. Clearly, if $u_{\max } \leq$ $\sqrt{\kappa_{3} / \kappa_{1}}$, then $E_{\mathrm{M}, \text { norm }, i}^{\star}=E_{\mathrm{M}, \text { norm }, j}^{\star}$. In summary, we have $E_{\mathrm{M}, \text { norm }, i}^{\star} \leq E_{\mathrm{M}, \text { norm }, j}^{\star}$ if $\mathbb{E}\left\{1 / \Upsilon_{i}\right\}<\mathbb{E}\left\{1 / \Upsilon_{j}\right\}$.

Corollary 2 shows that the robot should spend more motion energy per unit length to escape from the locations where the predicted channel qualities are lower. Also, it should spend more communication energy per unit length to take advantage of the locations where the predicted channel qualities are higher.

Remark 2: So far, we assumed that $\kappa_{1}, \kappa_{2}$ and $\kappa_{3}$ are positive constants. Experimental results have shown that a linear model $\left(\kappa_{1}=0\right)$ can also be used to approximate the motion energy cost for some types of robots, when the velocity is not very large [21]. Furthermore, there could be cases where $\kappa_{3}=0$. Theorem 2 can be easily extended to address these special cases as we show in the next two lemmas.

Lemma 4: Consider the case where $\kappa_{1}=0$ and $\kappa_{3} \neq 0$. The optimum motion speed $\left(u_{i}^{\star}\right)$, transmission rate $\left(R_{i}^{\star}\right)$ and stop time $\left(t_{\mathrm{st}, i}^{\star}\right)$ satisfy the following properties: $u_{i}^{\star}=u_{\max }$ for all $i$. If $\mathbb{E}\left\{1 / \Upsilon_{i}\right\}<\mathbb{E}\left\{1 / \Upsilon_{j}\right\}$, then $R_{i}^{\star} \geq R_{j}^{\star}$ for $i, j \in\left\{1, \cdots, N_{\mathrm{SH}}\right\}$. Moreover, if $\mathbb{E}\left\{1 / \Upsilon_{i}\right\}$ is above a certain threshold, then there is no transmission in the corresponding sub-trajectory $\left(R_{i}^{\star}=0\right)$. Finally, $t_{\mathrm{st}, i}^{\star}=T-L / u_{\max }$ if $\mathbb{E}\left\{1 / \Upsilon_{i}\right\}=\min _{j \in\left\{1, \cdots, N_{\mathrm{SH}}\right\}}\left\{\mathbb{E}\left\{1 / \Upsilon_{j}\right\}\right\}$. As compared to the results in Theorem 2 , it can be seen that the robot always travels with its maximum velocity in this case since the distance to be travelled is given. Then the robot spends as much time as possible at the location that has the best estimated channel quality. ${ }^{4}$

Lemma 5: Consider the case where $\kappa_{1} \neq 0$ and $\kappa_{3}=0$. The optimum motion speed $\left(u_{i}^{\star}\right)$, transmission rate $\left(R_{i}^{\star}\right)$ and stop time $\left(t_{\mathrm{st}, i}^{\star}\right)$ satisfy the following properties: if $\mathbb{E}\left\{1 / \Upsilon_{i}\right\}<\mathbb{E}\left\{1 / \Upsilon_{j}\right\}$, then $u_{i}^{\star} \leq u_{j}^{\star}$ and $R_{i}^{\star} \geq R_{j}^{\star}$ for $i, j \in\left\{1, \cdots, N_{\mathrm{SH}}\right\}$. Moreover, if $\mathbb{E}\left\{1 / \Upsilon_{i}\right\}$ is above a certain threshold, then there is no transmission in the corresponding sub-trajectory $\left(R_{i}^{\star}=0\right)$. Finally, $t_{\mathrm{st}, i}^{\star}=0$ for all $i$. As compared to Theorem 2, the robot chooses to reduce its velocity rather than stopping, in order to save motion energy in this case.

Corollary 2 can similarly be generalized for the two cases discussed above. In the rest of the paper, we assume that $\kappa_{1}$, $\kappa_{2}$ and $\kappa_{3}$ are positive constants.

\section{B. Two Special Cases - Cases of Heavy-Task Load and Light- Task Load}

In this section, we discuss two special cases of (2), namely the heavy-task load and the light-task load cases, in order to have a better understanding of the optimum design strategy.

Definition 2: We say that the robot has a heavy-task load if $Q / T$ is considerably large, i.e. it needs to send a large number of bits in a relatively small given time budget. On the other hand, the robot has a light-task load if $Q / T \rightarrow 0$, i.e. it only needs to send a small number of bits under a relatively large time budget.

Lemma 6: Let $k_{\text {best }}$ and $k_{\text {worst }}$ denote the indices where $\mathbb{E}\left\{1 / \Upsilon_{i}\right\}$ has its minimum and maximum based on the predicted channel respectively. If $u_{\max }>\sqrt{\kappa_{3} / \kappa_{1}}$ and $Q / T>$ $\max \left\{B\left(\log _{2}\left(\mathbb{E}\left\{1 / \Upsilon_{k_{\text {worst }}}\right\}\right)-\log _{2}\left(\mathbb{E}\left\{1 / \Upsilon_{k_{\text {best }}}\right\}\right)\right), \eta\right\}$, where $\eta=\max _{i \in\left\{1, \cdots, N_{\mathrm{SH}}\right\}}\left\{B \log _{2}\left(\left[K \kappa_{1} u_{\max }^{2}-\right.\right.\right.$ $\left.\mathbb{E}\left\{1 / \Upsilon_{k_{\text {best }}}\right\} \quad+\quad \mathbb{E}\left\{1 / \Upsilon_{i}\right\}\right] /\left[\left(\log _{2}\left(\mathbb{E}\left\{1 / \Upsilon_{i}\right\}\right) \quad-\right.\right.$ $\left.\left.\left.\left.\log _{2}\left(\mathbb{E}\left\{1 / \Upsilon_{k_{\text {best }}}\right\}\right)\right) \mathbb{E}\left\{1 / \Upsilon_{k_{\text {best }}}\right\} \ln (2)\right]\right)\right\}$, then we have: 1) $u_{i}^{\star}=u_{\max }$ for all $i \neq k_{\text {best }}$, and $u_{k_{\text {best }}}^{\star}=\max \left\{\sqrt{\kappa_{3} / \kappa_{1}}, l_{k_{\text {best }}} /\left(T-\sum_{i \neq k_{\text {best }}} l_{i} / u_{\max }\right)\right\}$; and 2) $t_{\mathrm{st}, k_{\text {best }}}^{\star}=\max \left\{T-\sum_{i \neq k_{\text {best }}} l_{i} / u_{\max }-l_{k_{\text {best }}} \sqrt{\kappa_{1} / \kappa_{3}}, 0\right\}$. Therefore, given an arbitrarily large $u_{\max }$ and an arbitrarily large $Q / T$, we have the following asymptotic behavior: 1) $u_{i}^{\star}=u_{\max }$ can become arbitrarily large for all $\left.i \neq k_{\text {best }} ; 2\right)$ $\left(t_{\text {mo }, k_{\text {best }}}^{\star}+t_{\text {st }, k_{\text {best }}}^{\star}\right) / T$ can become arbitrarily close to 1 ; and 3$)$ $R_{k_{\text {best }}}^{\star}\left(t_{\text {mo, } k_{\text {best }}}^{\star}+t_{\text {st }, k_{\text {best }}}^{\star}\right) /(Q / B)$ can become arbitrarily close to 1 .

Proof: From Theorem 2, it can be seen that $R_{k_{\text {best }}}^{\star}=$ $\log _{2}\left(K \lambda^{\star} / \ln (2)\right)-\log _{2}\left(\mathbb{E}\left\{1 / \Upsilon_{k_{\text {best }}}\right\}\right) \geq Q /(B T)$. This is lower bounded by $Q /(B T)$ since the robot has to send with at least as fast as the average rate $(Q /(B T))$ at the place with the best predicted channel quality, in order to finish the task in the given time. Hence, if $Q / T>B\left(\log _{2}\left(\mathbb{E}\left\{1 / \Upsilon_{k_{\text {worst }}}\right\}\right)-\right.$ $\left.\log _{2}\left(\mathbb{E}\left\{1 / \Upsilon_{k_{\text {best }}}\right\}\right)\right)$, we have $\mathbb{E}\left\{1 / \Upsilon_{i}\right\}<K \lambda^{\star} / \ln (2)$ for all

\footnotetext{
${ }^{4}$ Note that if $\kappa_{1}=\kappa_{3}=0$, Lemma 4 still holds except that the robot does not need to travel with its maximum speed along the sub-trajectory with the best estimated channel quality to achieve the optimality.
} 
$i$. From (3), we then have the following for $i \neq k_{\text {best }}$ :

$$
\begin{aligned}
& \kappa_{1} u_{i}^{\star 2}-\kappa_{1} u_{k_{\text {best }}}^{\star 2}=\log _{2}\left(\mathbb{E}\left\{\frac{1}{\Upsilon_{i}}\right\} / \mathbb{E}\left\{\frac{1}{\Upsilon_{k_{\text {best }}}}\right\}\right) \lambda^{\star} \\
& +\frac{1}{K}\left(\mathbb{E}\left\{\frac{1}{\Upsilon_{k_{\text {best }}}}\right\}-\mathbb{E}\left\{\frac{1}{\Upsilon_{i}}\right\}\right)-\epsilon_{i}^{\star}+\epsilon_{k_{\text {best }}}^{\star} \\
& \geq \log _{2}\left(\mathbb{E}\left\{\frac{1}{\Upsilon_{i}}\right\} / \mathbb{E}\left\{\frac{1}{\Upsilon_{k_{\text {best }}}}\right\}\right) \frac{\ln (2)}{K} 2^{Q /(B T)} \mathbb{E}\left\{\frac{1}{\Upsilon_{k_{\text {best }}}}\right\} \\
& +\frac{1}{K}\left(\mathbb{E}\left\{\frac{1}{\Upsilon_{k_{\text {best }}}}\right\}-\mathbb{E}\left\{\frac{1}{\Upsilon_{i}}\right\}\right)-\epsilon_{i}^{\star}+\epsilon_{k_{\text {best }}}^{\star} \\
& >\kappa_{1} u_{\text {max }}^{2}-\epsilon_{i}^{\star}+\epsilon_{k_{\text {best }}}^{\star},
\end{aligned}
$$

if $Q / T>\eta$. This equation implies that $\epsilon_{i}^{\star}>0$ for all $i \neq k_{\text {best }}$. Hence, we have $u_{i}^{\star}=u_{\max }$ for all $i \neq k_{\text {best }}$, and $u_{k_{\text {best }}^{\star}}^{\star}=\max \left\{\sqrt{\kappa_{3} / \kappa_{1}}, l_{k_{\text {best }}} /\left(T-\sum_{i \neq k_{\text {best }}} l_{i} / u_{\max }\right)\right\}$. Also, $t_{\text {st }, k_{\text {best }}}^{\star}=\max \left\{T-\sum_{i \neq k_{\text {best }}} l_{i} / u_{\max }-l_{k_{\text {best }}} \sqrt{\kappa_{1} / \kappa_{3}}, 0\right\}$. Therefore, given an arbitrarily large $u_{\max }$, the asymptotic behavior can be easily verified.

Intuitively, if the task load is heavy, it is more important for the robot to minimize the communication energy cost. Lemma 6 says that, in this case, the robot will spend as long time as possible at the location that has the best predicted channel quality in order to save communication energy. To achieve this, the robot needs to travel with its maximum velocity to pass other places quickly.

Lemma 7: Let $k_{\text {best }}$ denote the index where $\mathbb{E}\left\{1 / \Upsilon_{i}\right\}$ has its minimum based on the predicted channel. If $u_{\max }>$ $\sqrt{\kappa_{3} / \kappa_{1}}$ and $Q / T \rightarrow 0$, then 1) $u_{i}^{\star} \rightarrow u_{k_{\text {best }}}^{\star}$ and $R_{i}^{\star}=0$ for all $i \neq k_{\text {best }}$; 2) if $T \geq \sqrt{\kappa_{1} / \kappa_{3}} L$, then $u_{k_{\text {best }}}^{\star}=\sqrt{\kappa_{3} / \kappa_{1}}$ and $\left.t_{\text {st }, k_{\text {best }}}^{\star} \rightarrow T-\sqrt{\kappa_{1} / \kappa_{3}} L ; 3\right)$ if $T<\sqrt{\kappa_{1} / \kappa_{3}} L$, then $u_{k_{\text {best }}}^{\star} \rightarrow$ $L / T$ and $t_{\mathrm{st}, k_{\text {best }}}^{\star}=0$; and 4) $R_{k_{\text {best }}}^{\star}\left(t_{\mathrm{mo}, k_{\text {best }}}^{\star}+t_{\mathrm{st}, k_{\text {best }}}^{\star}\right) /(Q / B)=$ 1.

Proof: From Theorem 2, we know that the robot moves slower or even stops along $\mathcal{T}_{k_{\text {best }}}$ (the sub-trajectory with the best predicted channel quality). Hence, the time budget allocated to $\mathcal{T}_{k_{\text {best }}}$ should be larger than or equal to $T l_{k_{\text {best }}} / L$. Then, we have $R_{k_{\text {best }}}^{\star}=\log _{2}\left(K \lambda^{\star} / \ln (2)\right)-$ $\log _{2}\left(\mathbb{E}\left\{1 / \Upsilon_{k_{\text {best }}}\right\}\right) \leq Q /\left(B T l_{k_{\text {best }}} / L\right)$. Therefore, as $Q / T \rightarrow$ 0 , we have $\mathbb{E}\left\{1 / \Upsilon_{i}\right\}>K \lambda^{\star} / \ln (2)$ for all $i \neq k_{\text {best }}$. This means that $R_{i}^{\star}=0$ for all $i \neq k_{\text {best }}$, based on Theorem 2 . Similar to the proof of Lemma 6, we then have

$$
\begin{aligned}
& 0 \leq \kappa_{1} u_{i}^{\star 2}-\kappa_{1} u_{k_{\text {best }}}^{\star 2}=\frac{1}{K} \mathbb{E}\left\{\frac{1}{\Upsilon_{k_{\text {best }}}}\right\}-\left(\frac{1}{\ln (2)}-R_{k_{\text {best }}}^{\star}\right) \lambda^{\star} \\
& -\epsilon_{i}^{\star}+\epsilon_{k_{\text {best }}}^{\star} \leq \frac{1}{K} \mathbb{E}\left\{\frac{1}{\Upsilon_{k_{\text {best }}}}\right\}-\left(\frac{1}{\ln (2)}-\frac{L Q}{B T l_{k_{\text {best }}}}\right) \frac{\ln (2)}{K} \\
& 2^{Q /(B T)} \mathbb{E}\left\{\frac{1}{\Upsilon_{k_{\text {best }}}}\right\}-\epsilon_{i}^{\star}+\epsilon_{k_{\text {best }}}^{\star} \leq \frac{1}{K} \mathbb{E}\left\{\frac{1}{\Upsilon_{k_{\text {best }}}}\right\} \\
& -\left(\frac{1}{\ln (2)}-\frac{L Q}{B T l_{k_{\text {best }}}}\right) \frac{\ln (2)}{K} 2^{Q /(B T)} \mathbb{E}\left\{\frac{1}{\Upsilon_{k_{\text {best }}}}\right\}
\end{aligned}
$$

where the last inequality holds since $u_{i}^{\star} \geq u_{k_{\text {best }}}^{\star}$, resulting in $\epsilon_{i}^{\star} \geq \epsilon_{k_{\text {best }}}^{\star}$. As can be seen, as $Q / T \rightarrow 0$, the right hand side of (5) goes to 0 . Thus, $u_{i}^{\star} \rightarrow u_{k_{\text {best }}}^{\star}$ for all $i \neq k_{\text {best }}$. Also, from Theorem 2, we have $t_{\mathrm{st}, i}^{\star}=0$ for all $i \neq k_{\text {best }}$. Then, from Corollary 1, it is straightforward to show that $u_{k_{\text {best }}}^{\star}=\sqrt{\kappa_{3} / \kappa_{1}}$ and $t_{\mathrm{st}, k_{\text {best }}}^{\star} \rightarrow T-\sqrt{\kappa_{1} / \kappa_{3}} L$ if $T \geq \sqrt{\kappa_{1} / \kappa_{3}} L$, and $u_{k_{\text {best }}}^{\star} \rightarrow$ $L / T$ and $t_{\mathrm{st}, k_{\text {best }}}^{\star}=0$ if $T<\sqrt{\kappa_{1} / \kappa_{3}} L$. Finally, since $R_{i}^{\star}=0$ for all $i \neq k_{\text {best }}$, we must have $R_{k_{\text {best }}}^{\star}\left(t_{\mathrm{mo}, k}^{\star}+t_{\mathrm{st}, k_{\text {best }}}^{\star}\right) /(Q / B)=$ 1.

As compared to the heavy-task load case, it is more important to minimize the motion energy cost if the task load is light. Lemma 7 shows that in this case, the robot moves with an asymptotic constant speed of $\sqrt{\kappa_{3} / \kappa_{1}}$ along the whole trajectory in order to save motion energy. The constant speed is the speed that minimizes the motion energy if that speed can ensure achieving the task in the given time budget. If not, then the constant speed is $L / T$. Finally, since there is only very limited number of bits to be sent, the robot only transmits at the location with the best predicted channel quality.

Remark 3: Note that we only consider the case where $u_{\max }>\sqrt{\kappa_{3} / \kappa_{1}}$ in Lemmas 6 and 7. If $u_{\max } \leq \sqrt{\kappa_{3} / \kappa_{1}}$, then $u_{i}^{\star}=u_{\max }$ for all $i$, as we have shown in Corollary 1 .

\section{Stop-Time Online Adaptation to Multipath FADING}

As mentioned previously, the channel along each $\mathcal{T}_{i}$ is still space-varying due to multipath fading, especially in rich scattering environments. Unlike path loss and shadowing, the multipath fading component of the channel is unpredictable. Hence, there is no efficient way of predicting its spatial variations ahead of time and planning accordingly. However, as the robot moves along each sub-trajectory, it can measure the true value of the channel and further fine tune its strategies, as we propose in this section. More specifically, we show how the robot can optimize its stop time location within a designated sub-trajectory, based on its online measurement of the channel and by using the computationally-efficient nested form of multi-stage stochastic programming [22]. From Corollary 1, we know that the total motion time of the whole operation is always less than or equal to $\sqrt{\kappa_{1} / \kappa_{3}} L$. Then, the robot will spend most of its time resource on the stop time, if the time budget is considerably larger than $\sqrt{\kappa_{1} / \kappa_{3}} L$. Therefore, carefully selecting the location of the stop time within the designated sub-trajectory (which is found in Section III) can further reduce the energy consumption, as we show in this section.

Let $\mathcal{T}_{i}$ denote the sub-trajectory that was assigned a stop time based on (2). We divide $\mathcal{T}_{i}$ into $N_{\mathrm{MP}, i}$ equal-length chunks, $\mathcal{T}_{i, j}$ s, for $j \in\left\{1, \cdots, N_{\mathrm{MP}, i}\right\}$, over which the channel is considered constant. Let $\gamma_{i, j}$ denote the CNR in $\mathcal{T}_{i, j}$. The process of online adaptation along $\mathcal{T}_{i}$ is summarized as follows. The robot obtains the optimum number of bits of information, and the motion and stop times that are assigned to $\mathcal{T}_{i}$ from (2). As the robot moves to $\mathcal{T}_{i, 1}$, it measures $\gamma_{i, 1}$. Then, it fine tunes its strategy based on the measurement of $\gamma_{i, 1}$, and the estimation of $\gamma_{i, j}$ for $j \in\left\{2, \cdots, N_{\mathrm{MP}, i}\right\}$. This process will go on until it reaches the end of $\mathcal{T}_{i}$. For those CNR values that the robot has not observed yet, it models them with the probabilistic channel assessment framework of Section II-A, i.e. as lognormal random variables. More specifically, let $R_{i, j}, t_{\mathrm{mo}, i, j}$ and $t_{\mathrm{st}, i, j}$ be the spectral efficiency, motion time and stop time allocated to $\mathcal{T}_{i, j}$ respectively. Then, we have $R_{i, j}=R_{i}^{\star}$ and $t_{\mathrm{mo}, i, j}=t_{\mathrm{mo}, i}^{\star} / N_{\mathrm{MP}, i}$ for all $j$, and we need to design a stop time strategy subject to $\sum_{j=1}^{N_{\mathrm{MP}, i}} t_{\mathrm{st}, i, j}=t_{\mathrm{st}, i}^{\star}>0$, where $R_{i}^{\star}, t_{\mathrm{mo}, i}^{\star}$ and $t_{\mathrm{st}, i}^{\star}$ are given by the solution of (2). Thus, the variables to solve for are $t_{\mathrm{st}, i, j} \mathrm{~s}$. 
In this paper, we use multi-stage stochastic programming to design such a strategy [22]. In particular, we consider the nested form of the objective function, which allows us to solve the problem iteratively. Then, at step $\mathcal{T}_{i, j}$, for $j \in\left\{1, \cdots, N_{\mathrm{MP}, i}-1\right\}$, the robot can solve the following optimization problem to decide how to use the stop time:

$$
\begin{array}{cc}
\min & \mathcal{J}_{\mathrm{MP}, \mathrm{st}, i, j}\left(T_{\mathrm{st}, i, j}\right)=\frac{2^{R_{i}^{\star}}-1}{K \gamma_{i, j}}\left(\sqrt{\frac{\kappa_{1}}{\kappa_{3}}} \frac{l_{i}}{N_{\mathrm{MP}, i}}+t_{\mathrm{st}, i, j}\right) \\
& +\frac{2 \sqrt{\kappa_{1} \kappa_{3}} l_{i}}{N_{\mathrm{MP}, i}}+\mathbb{E}\left\{\mathcal{J}_{\mathrm{MP}, \mathrm{st}, i, j+1}^{\star}\left(T_{\mathrm{st}, i, j+1}\right)\right\} \\
\text { s.t. } & t_{\mathrm{st}, i, j}+T_{\mathrm{st}, i, j+1}=T_{\mathrm{st}, i, j}, \quad t_{\mathrm{st}, i, j}, T_{\mathrm{st}, i, j+1} \geq 0,
\end{array}
$$

where $T_{\mathrm{st}, i, j+1}=t_{\mathrm{st}, i}^{\star}-\sum_{\ell=1}^{j} t_{\mathrm{st}, i, \ell}^{\star}$ denotes the remaining stop time available at step $\mathcal{T}_{i, j+1}$, and $\mathcal{J}_{\mathrm{MP}, \mathrm{st}, i, j}$ is the average total energy cost from $\mathcal{T}_{i, j}$ to $\mathcal{T}_{i, N_{\mathrm{MP}, i}}$. Note that $T_{\mathrm{st}, i, 1}=t_{\mathrm{st}, i}^{\star}$, i.e. the remaining stop time at step $\mathcal{T}_{i, 1}$ is equal to the stop time that is assigned to $\mathcal{T}_{i}$. Also, we use the fact that $t_{\mathrm{mo}, i}^{\star}=$ $\sqrt{\kappa_{1} / \kappa_{3}} l_{i}$ in (6).

The optimization problem of (6) minimizes the average total energy cost along the rest of $\mathcal{T}_{i}$, i.e. from $\mathcal{T}_{i, j}$ to $\mathcal{T}_{i, N_{\mathrm{MP}, i}}$. Note that, at step $\mathcal{T}_{i, j}, \gamma_{i, j}$ is a constant since it has already been measured by the robot. Then the sum of the first and the second terms of the objective function in (6) represents the energy cost along $\mathcal{T}_{i, j}$. However, $\mathcal{J}_{\mathrm{MP}, \mathrm{st}, i, j+1}^{\star}$, which is a function of $\gamma_{i, \ell}$, for $\ell \in\left\{j+1, \cdots, N_{\mathrm{MP}, i}\right\}$, is still a random variable. Hence, we use the average of $\mathcal{J}_{\mathrm{MP}, \mathrm{tst}, i, j+1}^{\star}$ to represent the energy cost. The averaging is done over the distribution of the remaining unvisited channel samples in that sub-trajectory, which is characterized by using our probabilistic channel assessment framework.

Since solving the optimization problem at step $\mathcal{T}_{i, j}$ requires the average of the optimum value at step $\mathcal{T}_{i, j+1}$, i.e. $\mathbb{E}\left\{\mathcal{J}_{\mathrm{MP}, \mathrm{st}, i, j+1}^{\star}\left(T_{\mathrm{st}, i, j+1}\right)\right\}$, equation (6) can be solved from the $\left(N_{\mathrm{MP}, i}-1\right)^{\text {th }}$ step, and then backtracked to the $j^{\text {th }}$ step, which is similar to dynamic programming. Note that, at each step, equation (6) is a linear program, which can be solved uniquely. Then, we have the following optimum stop-time online adaptation strategy:

Lemma 8: The optimum stop-time online adaptation strategy along $\mathcal{T}_{i, j}$, for $j \in\left\{1, \cdots, N_{\mathrm{MP}, i}-1\right\}$, is (7). Moreover, the average total energy cost along $\mathcal{T}_{i}$, after implementing (7), is (8). Equations (7) and (8) are shown on the top of the next page. ${ }^{5}$

Proof: Consider the $\left(N_{\mathrm{MP}, i}-1\right)^{\text {th }}$ step of the optimization problem of (6). It can be shown that $t_{\mathrm{st}, i, N_{\mathrm{MP}, i}-1}^{\star}=\left\{\begin{array}{ll}T_{\mathrm{st}, i, N_{\mathrm{MP}, i}-1} & \text { if } 1 / \gamma_{i, N_{\mathrm{MP}, i}-1} \leq \mathbb{E}\left\{1 / \Upsilon_{i}\right\} \\ 0 & \text { otherwise }\end{array}\right.$.

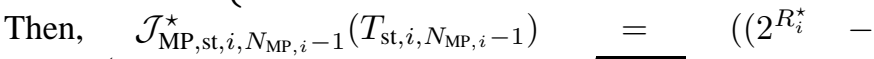
$1) / K)\left(\left(\mathbb{E}\left\{1 / \Upsilon_{i}\right\}+1 / \gamma_{i, N_{\mathrm{MP}, i}-1}\right) \sqrt{\kappa_{1} / \kappa_{3}} l_{i} / N_{\mathrm{MP}, i}+\right.$ $\left.\min \left\{1 / \gamma_{i, N_{\mathrm{MP}, i}-1}, \mathbb{E}\left\{1 / \Upsilon_{i}\right\}\right\} T_{\mathrm{st}, i, N_{\mathrm{MP}, i}-1}\right)+4 \sqrt{\kappa_{1} \kappa_{3}} l_{i} / N_{\mathrm{MP}, i}$. At the $\left(N_{\mathrm{MP}, i}-2\right)^{\text {th }}$ step, $\gamma_{i, N_{\mathrm{MP}, i}-1}$ is a random variable. Thus, we have the following averaging $\mathbb{E}\left\{\mathcal{J}_{\mathrm{MP}, \mathrm{st}, i, N_{\mathrm{MP}, i}-1}^{\star}\left(T_{\mathrm{st}, i, N_{\mathrm{MP}, i}-1}\right)\right\}=$ $\left(\left(2^{R_{i}^{\star}} \quad-1\right) / K\right)\left(2 \mathbb{E}\left\{1 / \Upsilon_{i}\right\} \sqrt{\kappa_{1} / \kappa_{3}} l_{i} / N_{\mathrm{MP}, i} \quad+\right.$ $\left.\mathbb{E}\left\{\min \left\{1 / \Upsilon_{i}, \mathbb{E}\left\{1 / \Upsilon_{i}\right\}\right\}\right\} T_{\mathrm{st}, i, N_{\mathrm{MP}, i}-1}\right)+4 \sqrt{\kappa_{1} \kappa_{3}} l_{i} / N_{\mathrm{MP}, i}$.

\footnotetext{
${ }^{5}$ Note that one-step nested expectation means $\mathbb{E}\left\{1 / \Upsilon_{i}\right\}$. Similarly, twostep nested expectation means $\mathbb{E}\left\{\min \left\{1 / \Upsilon_{i}, \mathbb{E}\left\{1 / \Upsilon_{i}\right\}\right\}\right\}$. The same rule applies to higher steps.
}

The $\left(N_{\mathrm{MP}, i}-2\right)^{\mathrm{th}}$ step of the optimization problem of (6) can be solved similarly. By induction, we can backtrack to the $j^{\text {th }}$ step. Equations (7) and (8) can then be easily confirmed in this way.

The optimum strategy of (7) results in the robot only stopping once along $\mathcal{T}_{i}$ to spend all its time budget. The lemma then allows the robot to optimally choose that stop location. More specifically, the nested expectation in (7) decreases as the number of nested steps increases. Hence, the nested expectation is small when the robot operates at the beginning of $\mathcal{T}_{i}$, i.e. when $j$ is small. This means that the robot will spend all the stop time resource at the beginning of $\mathcal{T}_{i}$ only if the measured value of $\gamma_{i, j}$ is very large. On the other hand, the nested expectation becomes larger towards the end of $\mathcal{T}_{i}$, i.e. when $j$ is large. This implies that the robot is more likely to choose a location to stop if it is towards the end of the subtrajectory and it has not found a good spot yet. Intuitively, at the beginning of $\mathcal{T}_{i}$, the robot is not in a rush to spend the stop time since there is a good chance that a better $\gamma_{i, j}$ can be observed later. However, as the robot approaches the end of $\mathcal{T}_{i}$, the probability of observing a better $\gamma_{i, j}$ becomes smaller, forcing the robot to choose the stop location quicker.

Note that, for a lognormally-distributed $\Upsilon_{i}$, the nested expectation can be found by using (9), shown on the top of the next page, iteratively, where $\varphi$ is a constant calculated from the previous iteration (the previous nested expectation) and $\Theta$ is the $\mathrm{Q}$ function. To reduce the computation, the nested expectation can be found offline before the operation and saved to the memory of the robot.

In summary, the following are the steps of our proposed co-optimization framework. First, the robot predicts the shadowing and path loss components of the channel along the given trajectory, based on a small number of a priori channel measurements in the same environment. Then, based on the given time budget, the total number of bits of information to be transmitted, and the required target BER, the robot finds the optimum motion speed, stop time, and transmission rate for each sub-trajectory, $\mathcal{T}_{i}$, by solving (2). If $t_{\mathrm{st}, i}^{\star}>0$, for some $i$, i.e. there is a stop time, the robot evaluates (9) iteratively for that corresponding sub-trajectory, and implements (7) based on the online measurement of the channel, in order to optimally choose the stop location.

\section{Simulation Results}

In this section, we test our framework by using real channel measurements from downtown San Francisco (data courtesy of W. M. Smith) [23]. ${ }^{6}$ Fig. 2 (a) shows the channel power along a fixed path with the length of $240 \mathrm{~m}$. As can be seen, the channel is dominated by shadowing and multipath fading. This waveform is taken from the real measurements of [23]. Since we do not know the transmit power in that specific measurement, we assume that $1 \mathrm{~W}$ of transmit power was used (without loss of generality) to generate the channel power waveform. This will serve as the true channel power for testing our framework in this section. Furthermore, we assume that the receiver noise power is $-80 \mathrm{dBm}$, and the robot has $10 \%$ a priori channel samples gathered in the same environment to

\footnotetext{
${ }^{6}$ Similar results are obtained with simulated channels
} 


$$
t_{\mathrm{st}, i, j}^{\star}= \begin{cases}T_{\mathrm{st}, i, j} & \text { if } \frac{1}{\gamma_{i, j}} \leq \underbrace{\mathbb{E}\left\{\min \left\{\frac{1}{\Upsilon_{i}}, \cdots, \mathbb{E}\left\{\min \left\{\frac{1}{\Upsilon_{i}}, \mathbb{E}\left\{\frac{1}{\Upsilon_{i}}\right\}\right\}\right\} \cdots\right\}\right\}}_{\left(N_{\mathrm{MP}, i}-j\right) \text {-step nested expectation }} . \\ 0 \quad \text { otherwise. }\end{cases}
$$

$$
\mathbb{E}\left\{\mathcal{J}_{\mathrm{MP}, \mathrm{st}, i, 1}^{\star}\left(t_{\mathrm{st}, i}^{\star}\right)\right\}=\frac{2^{R_{i}^{\star}}-1}{K}(\mathbb{E}\left\{\frac{1}{\Upsilon_{i}}\right\} \sqrt{\frac{\kappa_{1}}{\kappa_{3}}} l_{i}+\underbrace{\mathbb{E}\left\{\min \left\{\frac{1}{\Upsilon_{i}}, \cdots, \mathbb{E}\left\{\min \left\{\frac{1}{\Upsilon_{i}}, \mathbb{E}\left\{\frac{1}{\Upsilon_{i}}\right\}\right\}\right\} \cdots\right\}\right\}}_{N_{\mathrm{MP}, i} \text {-step nested expectation }} t_{\mathrm{st}, i}^{\star})+2 \sqrt{\kappa_{1} \kappa_{3}} l_{i}
$$

$$
\mathbb{E}\left\{\min \left\{\frac{1}{\Upsilon_{i}}, \varphi\right\}\right\}=\varphi \Theta\left(\frac{1}{\sqrt{\Sigma_{i}}} \frac{10}{\ln 10} \ln \left(\varphi \bar{\Upsilon}_{i}\right)\right)+\frac{1}{\bar{\Upsilon}_{i}} \exp \left(\left(\frac{\ln 10}{10}\right)^{2} \frac{\Sigma_{i}}{2}\right) \Theta\left(-\frac{1}{\sqrt{\Sigma_{i}}} \frac{10}{\ln 10} \ln \left(\varphi \bar{\Upsilon}_{i}\right)+\frac{\ln 10}{10} \sqrt{\Sigma_{i}}\right)
$$

estimate the channel. We take $l_{i}=10 \mathrm{~m}\left(N_{\mathrm{SH}}=24\right)$ for all $i$. Fig. 2 (b) then shows the estimated $\mathbb{E}\left\{1 / \Upsilon_{i}\right\}$, which is an indication of predicated channel quality along the fixed path (see Remark 1). Moreover, the motion parameters are taken as $u_{\max }=6 \mathrm{~m} / \mathrm{s}, \kappa_{1}=4.39, \kappa_{2}=24.67$ and $\kappa_{3}=14.77$ [24].

Fig. 2 shows the results of our co-optimization framework of Section III (only adaptation to shadowing and path loss). We have $Q / B=250 \mathrm{bits} / \mathrm{Hz}$ and $T=120$ seconds in this example. The optimization problem of (2) is solved numerically by using the Optimization Toolbox of MATLAB. As can be seen from Fig. 2 (c and d), the robot moves slower and sends faster at the places that have better predicted channel qualities. Moreover, the robot stops at the location with the best predicted channel quality, as marked in Fig. 2 (e). We next compare the total energy consumption with the two cases of 1) no planning, i.e. the robot travels with the constant speed of $L / T$ and chooses the constant spectral efficiency of $Q /(B T)$, and 2) separately optimizing the motion speed and the spectral efficiency, i.e. the robot travels with the constant speed of $L / T$ and optimizes the spectral efficiency based on the predicted channel quality. As compared to our co-optimization strategy, the robot needs to consume 2.06 times more energy in the case of no planning, and 1.76 times more in the case of separate optimization of motion speed and spectral efficiency in order to accomplish the same task. With the additional online stop-time adaptation of Section IV, the robot further optimizes where to stop in the designated sub-trajectory as it learns the true value of the channel along its path. In this example, this additional optimization can further save $18.4 \%$ energy as compared to the case of only implementing our co-optimization framework of Section III. Next, Fig. 3 and 4 show the optimum strategies of the heavy and light-task cases. We have $T=200$ seconds for both cases, $Q / B=1000$ bits $/ \mathrm{Hz}$ for the heavy-task load case and $Q / B=10 \mathrm{bits} / \mathrm{Hz}$ for the light-task load case. ${ }^{7}$ As can be seen in Fig. 3 (c), if

\footnotetext{
${ }^{7}$ We choose a small value for $Q / B$ to show the asymptotic behavior of the light-task load case. As such, the optimum solution is not exactly the same as the asymptotic case. But they are very close.
}

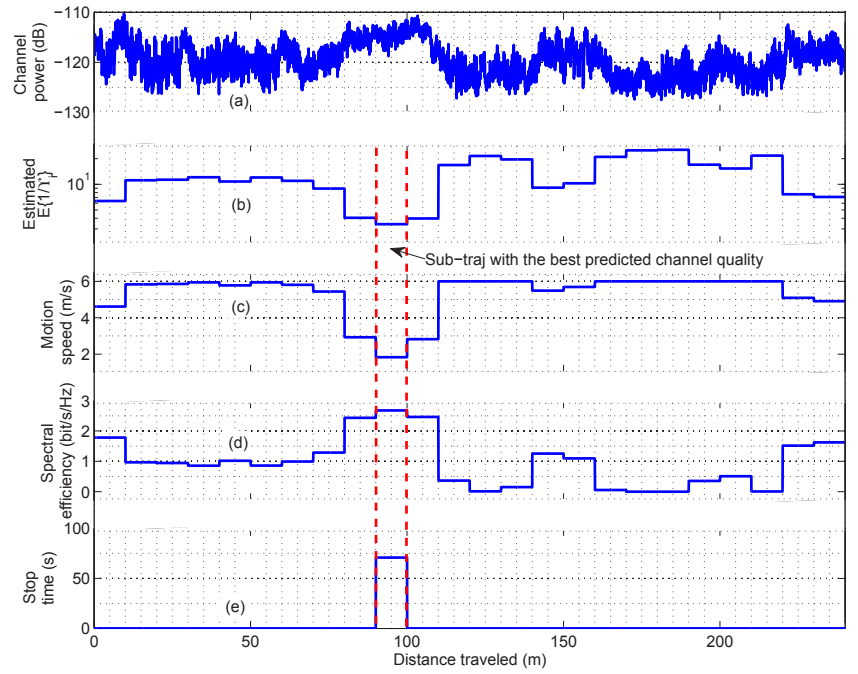

Fig. 2. Illustration of the performance of our co-optimization strategy. Figures $\mathrm{a}$ and $\mathrm{b}$ show the signal strength and the predicted channel quality along the trajectory respectively. Figures c, d and e show the optimum motion speed, spectral efficiency, and stop time of our proposed co-optimization framework of Section III respectively. In this case, the robot predicts the shadowing and path loss components of the channel along its trajectory and plans its strategy accordingly.

the robot has a heavy-task load, it moves with its maximum velocity along the sub-trajectories where the estimated channel qualities are not the best. The rest of the time budget (154 seconds) is allocated to the place with the best predicted channel quality. On the other hand, if the task load is light (Fig. 4), the robot moves with an almost constant speed along the whole trajectory and transmits at the location with the best predicted channel quality, as shown in Fig. 4 (c and d) respectively. Note that $u=\sqrt{\kappa_{3} / \kappa_{1}}=1.83 \mathrm{~m} / \mathrm{s}$ minimizes the motion energy cost along $\mathcal{T}$. It can be seen from Fig. 4 (c) that the robot moves with a speed very close to this value for the light-load case, as predicted by Lemma 7 .

\section{EXTENSIONS To ONLINE SENSING AND DATA GATHERING}

So far, we have considered the case where the bits of information that need to be transmitted are fixed and assigned 


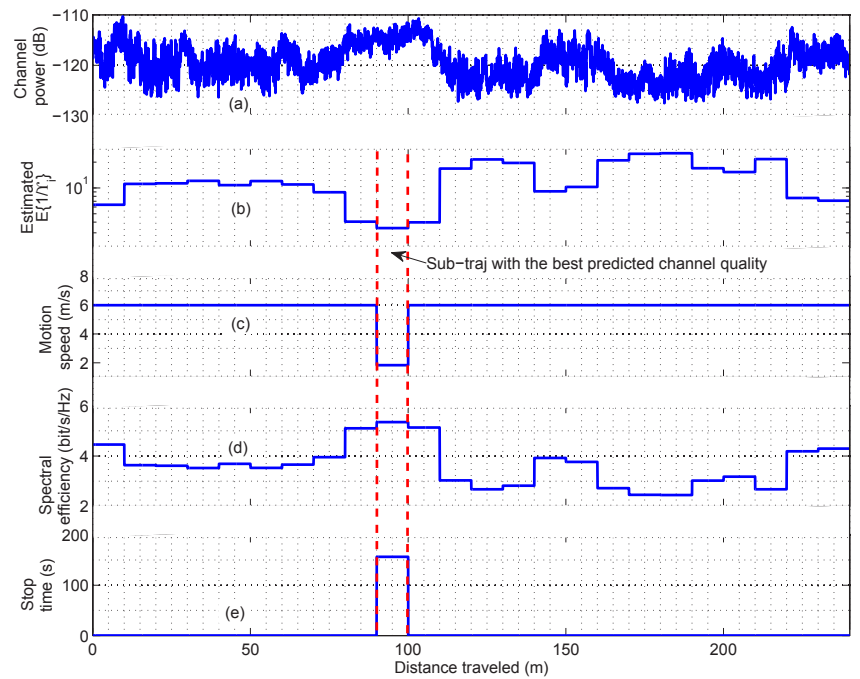

Fig. 3. Illustration of the performance of our co-optimization strategy for the case of heavy-task load.

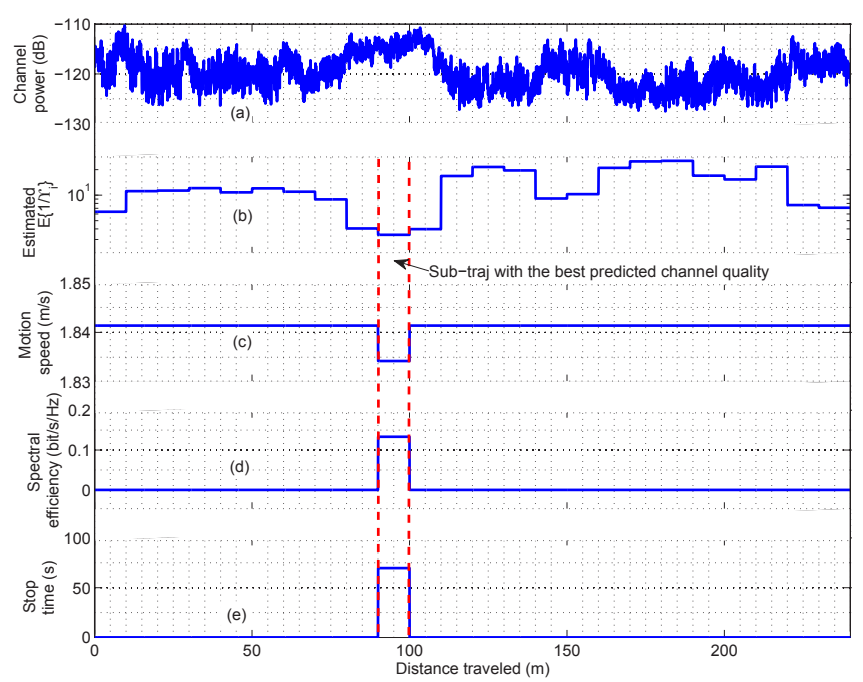

Fig. 4. Illustration of the performance of our co-optimization strategy for the case of light-task load.

to the robot at the beginning of the operation. In some applications, the robot is required to sense a number of points of interest, gather the sensing data, and then transmit the data to the remote station online and during the operation. In this part, we briefly discuss how our proposed co-optimization framework of Section III can be generalized to such cases. ${ }^{8}$

Consider the scenario where there are $N_{\text {I }}$ points of interest located at $z_{i} \in \mathcal{T}$, for $i \in\left\{1, \cdots, N_{\mathrm{I}}\right\}$, that the robot needs to visit. $\mathcal{T}$ is the pre-defined trajectory as defined previously. Let $Q_{i}>0$ denote the number of bits of information that the robot can gather at $z_{i}$. Also, let $Q_{0} \geq 0$ denote the initial number of bits of information that the robot has before starting the operation. Similar to Section III, $\mathcal{T}$ is divided into $N_{\mathrm{SH}}$ sub-trajectories $\left(\mathcal{T}_{i} \mathrm{~s}\right.$, for $\left.i \in\left\{1,2, \cdots, N_{\mathrm{SH}}\right\}\right)$. Without loss of generality, we also assume that $\mathcal{T}$ is divided in such a way that $z_{i}$ is located at the beginning of $\mathcal{T}_{\operatorname{Ind}\left(z_{i}\right)}$, where $\operatorname{Ind}\left(z_{i}\right)$ represents the index of the sub-trajectory where $z_{i}$ is located. Then, the optimization framework of (2) can be generalized

\footnotetext{
${ }^{8}$ The additional online adaptation of Section IV can be readily applied to this case. We therefore only focus on extending the results of Section III in this part.
}

as follows:

$$
\begin{array}{ll}
\min \quad & \mathcal{J}_{\mathrm{SH}}=\sum_{i=1}^{N_{\mathrm{SH}}} \frac{2^{R_{i}}-1}{K} \mathbb{E}\left\{\frac{1}{\Upsilon_{i}}\right\}\left(t_{\mathrm{mo}, i}+t_{\mathrm{st}, i}\right) \\
& +\frac{\kappa_{1} l_{i}^{2}}{t_{\mathrm{mo}, i}}+\kappa_{3} t_{\mathrm{mo}, i} \\
& \\
\text { s.t. } \quad & \sum_{i=1}^{N_{\mathrm{SH}}} t_{\mathrm{mo}, i}+t_{\mathrm{st}, i} \leq T, \\
& \sum_{i=1}^{N_{\mathrm{SH}}} R_{i}\left(t_{\mathrm{mo}, i}+t_{\mathrm{st}, i}\right)=\sum_{i=0}^{N_{\mathrm{I}}} Q_{i} / B, \\
& \operatorname{Ind}\left(z_{j}\right)-1 \\
& \sum_{i=1} R_{i}\left(t_{\mathrm{mo}, i}+t_{\mathrm{st}, i}\right) \leq \sum_{i=0}^{j} Q_{i} / B, \\
& \left.\forall j \in\left\{1, \cdots, N_{\mathrm{I}}\right\}, \quad R_{i}, t_{\mathrm{st}, i \geq 0,} \geq 0, N_{\mathrm{SH}}\right\} . \\
& t_{\mathrm{mo}, i} \geq l_{i} / u_{\mathrm{max}}, \forall i \in\{1, \cdots, \forall
\end{array}
$$

Note that, from $\mathcal{T}_{1}$ to $\mathcal{T}_{\operatorname{Ind}\left(z_{j}\right)-1}$, the total number of bits that can be gathered by the robot is $\sum_{i=0}^{j-1} Q_{i}$. Thus, the robot cannot send more information than it has gathered so far, which results in the third constraint in (10). Similar to Lemma 3 , it can also be shown that the constraints of the optimization problem of (10) satisfy LICQ at the optimum point. Hence, we have the following properties for the optimum motion and communication co-optimization strategy:

Theorem 3: The optimum motion speed $\left(u_{i}^{\star}\right)$, transmission rate $\left(R_{i}^{\star}\right)$ and stop time $\left(t_{\mathrm{st}, i}^{\star}\right)$ of (10) satisfy the following properties: if $\mathbb{E}\left\{1 / \Upsilon_{i}\right\}<\mathbb{E}\left\{1 / \Upsilon_{j}\right\}$ and there is no point of interest between $\mathcal{T}_{i}$ and $\mathcal{T}_{j}$, then $u_{i}^{\star} \leq u_{j}^{\star}$ and $R_{i}^{\star} \geq R_{j}^{\star}$, where $i, j \in\left\{1, \cdots, N_{\mathrm{SH}}\right\}$. Moreover, the robot may stop in more than one sub-trajectory during the operation. Let $\mathcal{K}_{\text {best }}$ denote the collective set of indices where $\mathbb{E}\left\{1 / \Upsilon_{i}\right\}$ has its minimum in the following intervals: from $\mathcal{T}_{1}$ to $\mathcal{T}_{\operatorname{Ind}\left(z_{1}\right)-1}$, from $\mathcal{T}_{\operatorname{Ind}\left(z_{j}\right)}$ to $\mathcal{T}_{\operatorname{Ind}\left(z_{j+1}\right)-1}$, for $j \in\left\{1, \cdots, N_{\mathrm{I}}-1\right\}$, and from $\mathcal{T}_{\operatorname{Ind}\left(z_{N_{\mathrm{I}}}\right)}$ to $\mathcal{T}_{N_{\mathrm{SH}}}$. Then, if $t_{\mathrm{st}, i}^{\star}>0$, we have $i \in \mathcal{K}_{\text {best }}$.

Proof: It is straightforward to extend the proof of Theorem 2 to this case. The details of the proof are omitted due to page limitation.

Similar to Theorem 2, Theorem 3 says that the robot moves slower and transmits faster along $\mathcal{T}_{i}$, as compared to $\mathcal{T}_{j}$, if the predicted channel quality along $\mathcal{T}_{i}$ is better and there is no point of interest between them. If there exists any point of interest between $\mathcal{T}_{i}$ and $\mathcal{T}_{j}$, and $j<i$, the same conclusion still holds. However, if $j>i$, then the robot may need to reduce its speed and transmit with a higher rate at $\mathcal{T}_{j}$ in order to ensure the transmission of the newly-gathered information. Also, the robot may stop at more than one location along the trajectory in order to spend long enough time for transmission. Theorem 3 says that, in such cases, the corresponding subtrajectories must have the best predicted channel qualities in their local areas, where each local area is an area that contains no point of interest.

\section{CONCLUSIONS}

In this paper, we considered the case where a robot is tasked with sending a number of given bits of information to a remote station, as it travels along a pre-defined trajectory and in a given time budget. We showed how the robot can co-plan its motion and communication strategies in order to minimize 
its total energy consumption (including both the motion and communication costs). More specifically, our results indicated that in order to save energy, the robot should move faster (slower) and send less (more) bits at the locations that have worse (better) predicted channel qualities. We furthermore proved that if the robot must stop, it should then stop only once and at the spot with the best predicted channel quality. We also proved some properties for two special cases of heavy and light-task loads. We then proposed an additional stoptime online adaptation strategy to further fine tune the stop location as the robot moves along its trajectory and measures the true value of the channel. Finally, we briefly discussed how our framework can be generalized to the case of online sensing and data gathering. Our simulation results showed that our proposed framework results in a considerable performance improvement.

\section{REFERENCES}

[1] Y. Yan and Y. Mostofi, "Co-optimization of communication and motion planning of a robotic operation in fading environments," in Asilomar Conf. Signals, Syst. Comput., Asilomar, CA, Nov. 2011.

[2] W. Wang, V. Srinivasan, B. Wang, and K. Chua, "Coverage for target localization in wireless sensor networks," IEEE Trans. Wireless Commun., vol. 7, no. 2, pp. 667-676, Feb. 2008

[3] F. Bullo, E. Frazzoli, M. Pavone, K. Savla, and S. L. Smith, "Dynamic vehicle routing for robotic systems," Proc. IEEE, vol. 99, no. 9, pp. 1482-1504, Sept. 2011.

[4] M. M. Zavlanos, M. B. Egerstedt, Y. C. Hu, and G. J. Pappas, "Graphtheoretic connectivity control of mobile robot networks," Proc. IEEE vol. 99, no. 9, pp. 1525-1540, July 2011.

[5] M. M. Zavlanos and G. J. Pappas, "Potential fields for maintaining connectivity of mobile networks," IEEE Trans. Robotics, vol. 23, no. 4, pp. 812-816, Aug. 2007.

[6] Y. Mostofi, "Decentralized communication-aware motion planning in mobile networks: An information-gain approach," J. Intelligent Robotic Syst., Special Issue Unmanned Autonomous Vehicles, vol. 56, no. 2, pp. 233-256, 2009.

[7] M. M. Zavlanos, A. Ribeiro, and G. J. Pappas, "Mobility and routing control in networks of robots," in Proc. IEEE Conf. Decision Control, Atlanta, GA, Dec. 2010, pp. 7545-7550.

[8] J. Fink, A. Ribeiro, and V. Kumar, "Motion planning for robust wireless networking," in Proc. IEEE International Conf. Robotics Automation, Saint Paul, MN, May 2012.

[9] A. Ghaffarkhah and Y. Mostofi, "Communication-aware motion planning in mobile networks," IEEE Trans. Automatic Control, Special Issue Wireless Sensor Actuator Netw., vol. 56, no. 10, pp. 2478-2485, 2011.

[10] M. Lindhé and K. H. Johanson, "Using robot mobility to exploit multipath fading," IEEE Wireless Commun., vol. 16, no. 1, pp. 30-37, Feb. 2009.

[11] C. C. Ooi and C. Schindelhauer, "Minimal energy path planning for wireless robots," Mobile Netw. Appl., vol. 14, no. 3, pp. 309-321, Jan. 2009.

[12] A. J. Goldsmith and S.-G. Chua, "Variable-rate variable-power MQAM for fading channels," IEEE Trans. Commun., vol. 45, no. 10, pp. 12181230, Oct. 1997.

[13] Y. Mei, Y.-H. Lu, Y. C. Hu, and C. S. G. Lee, "Energy-efficient motion planning for mobile robots," in Proc. IEEE Internationl Conf. Robotics Automation, New Orleans, LA, Apr. 2004, pp. 4344-4349.

[14] Y. Yan and Y. Mostofi, "Robotic router formation in realistic communication environments," IEEE Trans. Robotics, vol. 28, no. 4, pp. 810-827, Aug. 2012.
[15] Y. Mostofi, M. Malmirchegini, and A. Ghaffarkhah, "Estimation of communication signal strength in robotic networks," in Proc. 50th IEEE Int'l Conf. Robotics Automation, Anchorage, AK, May 2010, pp. 19461951.

[16] M. Malmirchegini and Y. Mostofi, "On the spatial predictability of communication channels," IEEE Trans. Wireless Commun., vol. 11, no. 3, pp. 964-978, Mar. 2012.

[17] S. L. Smith, M. Schwager, and D. Rus, "Persistent robotic tasks: Monitoring and sweeping in changing environments," IEEE Trans. Robotics, vol. 28, no. 2, pp. 410-426, Apr. 2012.

[18] A. Ghaffarkhah, Y. Yan, and Y. Mostofi, "Dynamic coverage of timevarying environments using a mobile robot - a communication-aware perspectiveg," in Proc. IEEE Globecom Workshop Wireless Netw. Unmanned Autonomous Vehicles, Huston, TX, Dec. 2011, pp. 1297-1302.

[19] A. Goldsmith, Wireless Communications. Cambridge University Press, 2005.

[20] J. N. S. J. Wright, Numerical Optimization, 2nd ed. Springer, 2006.

[21] Y. Mei, Y.-H. Lu, Y. C. Hu, and C. S. G. Lee, "Deployment of mobile robots with energy and timing constraints," IEEE Trans. Robotics, vol. 22, no. 3, pp. 507-522, June 2006.

[22] A. Shapiro, D. Dentcheva, and A. Ruszczynski, Lectures on Stochastic Programming: Modeling and Theory. SIAM-Society for Industrial and Applied Mathematics, 2009.

[23] W. M. Smith, Urban Propagation Modeling for Wireless Systems. $\mathrm{PhD}$ thesis, 2004.

[24] P. Tokekar, N. Karnad, and V. Isler, "Energy optimal velocity profiles for car-like robots," 2009, technical report.

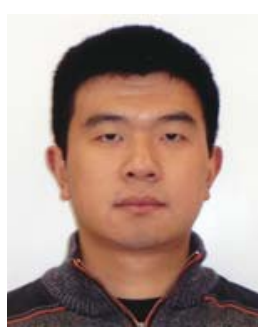

Yuan Yan received the BS and MS degrees from Huazhong University of Science and Technology, Wuhan, China, in 2006 and 2008, respectively. He is currently working towards his $\mathrm{PhD}$ degree in the Department of Electrical and Computer Engineering at the University of California Santa Barbara. His research interests include robotic router networks and communication-aware motion planning.

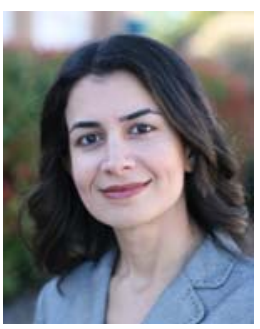

Yasamin Mostofi received the BS degree in electrical engineering from the Sharif University of Technology, Tehran, Iran, in 1997, and the MS and $\mathrm{PhD}$ degrees in the area of wireless communication systems from Stanford University, California, in 1999 and 2004, respectively. She is currently an associate professor in the Department of Electrical and Computer Engineering at the University of California Santa Barbara.

Dr. Mostofi is the recipient of the Presidential Early Career Award for Scientists and Engineers (PECASE), the US National Science Foundation (NSF) CAREER award, and the 2012 IEEE Region 6 Outstanding Engineer award. She also received the Bellcore fellow-advisor award from the Stanford Center for Telecommunications in 1999, the 2008- 2009 Electrical and Computer Engineering Distinguished Researcher Award from the University of New Mexico, and the 2012 IEEE Outstanding Engineer award of Albuquerque. Her current research lies at the intersection of the two areas of communications and control/robotics in mobile sensor networks. Current research projects include communication-aware navigation and decision making in robotic networks, compressive sensing and control, obstacle mapping, robotic routers, and cooperative information processing. She has served on the Control Systems Society conference editorial board since 2008. She is a member of the IEEE. 